\title{
Active Region Modulation of Coronal Hole Solar Wind
}

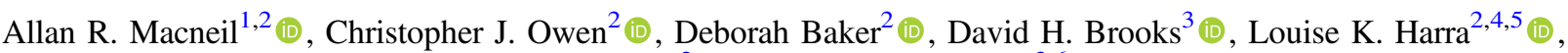 \\ David M. Long ${ }^{2}$ (D), and Robert T. Wicks ${ }^{2,6}$ (I) \\ ${ }^{1}$ Department of Meteorology, University of Reading, Reading, UK; a.r.macneil@ reading.ac.uk \\ ${ }^{2}$ Mullard Space Science Laboratory, University College London, Surrey, UK \\ ${ }^{3}$ College of Science, George Mason University, Fairfax, VA 22030, USA \\ ${ }_{5}^{4}$ Physikalisch Meteorologisches Observatorium Davos, World Radiation Center, Davos, Switzerland \\ 5 Department of Physics, Eidgenössische Technische Hochschule (ETH) Zurich, Zurich, Switzerland \\ ${ }^{6}$ Institute for Risk and Disaster Reduction, University College London, London, UK \\ Received 2019 July 23; revised 2019 October 22; accepted 2019 November 6; published 2019 December 17
}

\begin{abstract}
Active regions (ARs) are a candidate source of the slow solar wind (SW), the origins of which are a topic of ongoing research. We present a case study that examines the processes by which SW is modulated in the presence of an AR in the vicinity of the SW source. We compare properties of SW associated with a coronal hole (CH)quiet Sun boundary to SW associated with the same $\mathrm{CH}$ but one Carrington rotation later, when this region bordered the newly emerged NOAA AR 12532. Differences found in a range of in situ parameters are compared between these rotations in the context of source region mapping and remote sensing observations. Marked changes exist in the structure and composition of the SW, which we attribute to the influence of the AR on SW production from the $\mathrm{CH}$ boundary. These unique observations suggest that the features that emerge in the AR-associated wind are consistent with an increased occurrence of interchange reconnection during SW production, compared with the initial quiet Sun case.
\end{abstract}

Unified Astronomy Thesaurus concepts: Solar coronal holes (1484); Slow solar wind (1873); Solar active regions (1974); Solar magnetic reconnection (1504); Solar wind (1534); Heliosphere (711); Interplanetary magnetic fields (824); Solar atmosphere (1477)

\section{Introduction}

The processes by which the solar wind (SW) escapes into interplanetary space and is accelerated are still poorly understood. Knowledge of such processes is critical to our understanding of how the heliosphere is created. With the recent launch of NASA's Parker Solar Probe (Fox et al. 2016) and the upcoming launch of ESA's Solar Orbiter (Müller \& St. Cyr 2013), studies that gain insight into SW origins through combined in situ and remote sensing observation are particularly timely.

The contrasting properties of the "fast" and "slow" SW suggest that the two have different origins. Fast SW at 1 au exhibits bulk speeds $v \gtrsim 400 \mathrm{~km} \mathrm{~s}^{-1}$ and plasma number densities $n \sim 3 \mathrm{~cm}^{-3}$ (Schwenn 2007) and is relatively steady, except for Alfvénic fluctuations (Bame et al. 1977). Since early observations by Krieger et al. (1973), coronal holes (CHs) have been thought to be the source of the fast SW. Compositionally, fast SW features low ion charge states (Hundhausen et al. 1968; Owocki et al. 1983), which are consistent with the low electron temperature of a CH source (e.g., Feldman et al. 1999). Fast SW elemental abundances are also consistent with those of $\mathrm{CHs}$, in that they are not subject to strong "first ionization potential (FIP) bias." FIP bias is the enhancement in abundance (by a factor of $>2)$ of elements with low $(\lesssim 10 \mathrm{eV})$ FIP, which is observed in closed coronal loops (Meyer 1985; Laming et al. 1995; Brooks \& Warren 2011).

Slow SW $\left(v \lesssim 400 \mathrm{~km} \mathrm{~s}^{-1}\right)$ is often denser $\left(n \sim 10.7 \mathrm{~cm}^{-3}\right)$ and more variable and structured than fast SW (e.g., Bame et al. 1977; Schwenn et al. 1990; Schwenn 2007; Kepko et al. 2016). Slow wind minor ion composition features higher charge states than fast (e.g., Zhao et al. 2009) and strong FIP bias (Geiss et al. 1995). Slow wind plasma thus originates in closed-field regions but comes to escape into the heliosphere. Slow wind origins are therefore considerably less well known than fast ones and have been the focus of much prior and contemporary research (e.g., Wang \& Sheeley 1990; Geiss et al. 1995; Wang et al. 1996; Fisk et al. 1998; Antiochos et al. 2011; Riley \& Luhmann 2012; Brooks et al. 2015; Kepko et al. 2016; Owens et al. 2018). The release of slow wind plasma from coronal loops could be due to "interchange reconnection," i.e., reconnection between an open flux element and a closed loop (see Crooker et al. 2002). Interchange reconnection can occur at separatrices, or "quasi-separatrix layers" (QSLs, regions of rapidly changing connectivity; Priest \& Démoulin 1995). A range of prominent models for the origins of slow SW hinge on interchange reconnection (see Fisk et al. 1998; Schwadron et al. 1999; Fisk 2003; Antiochos et al. 2007, 2011).

\subsection{Active Regions (ARs) as SW Sources}

A host of studies have linked in situ SW observations to AR sources (Kojima et al. 1999; Neugebauer et al. 2002; Culhane et al. 2014; Fazakerley et al. 2016; Kilpua et al. 2016; Fu et al. 2017). Neugebauer et al. (2002) found AR-associated SW to exhibit moderately lower speeds, higher charge states, and greater variability in composition and plasma parameters than those from CHs, similarly to the slow wind. Fu et al. (2017) reported $42.9 \%$ of slow SW $\left(v<500 \mathrm{~km} \mathrm{~s}^{-1}\right)$ to be associated with an AR source. The study of ARs as SW sources thus appears crucial in shaping our understanding of the origins of particularly the slow SW.

AR SW has been studied extensively through remote observations, particularly from Hinode-EIS (Culhane et al. 2007; Kosugi et al. 2007). Continuous outflows have been 
observed from the edges of ARs (Sakao et al. 2007; Harra et al. 2008) located close to open magnetic field lines (as determined through potential field source surface [PFSS] modeling; Schatten et al. 1969), suggesting that this plasma is in fact able to escape into the SW. (In this study, we specifically define "outflowing" plasma to be upflowing plasma, which has access to the SW via open field lines.) These signatures were later found to be relatively common, but not ubiquitous (Edwards et al. 2016). Brooks \& Warren (2011) provided in situ confirmation of AR SW by linking highly fractionated outflowing regions at the edge of an AR observed with EIS to highly fractionated in situ SW observations. Furthermore, Brooks et al. (2015) combined EIS full-Sun spectroscopic velocity and composition observations with magnetic topology, and identified source regions where strongly FIP-biased plasma was outflowing with a total mass contribution sufficient to provide a significant fraction of the SW mass-loss rate. The highly fractionated plasma being located near an outflowing region is key, due to the often strong spatial variability in AR plasma composition (e.g., Baker et al. 2013; Brooks et al. 2015).

A range of mechanisms have been suggested by which SW might emerge from ARs. We shall now introduce three of these. First, interchange reconnection is found to commonly occur near ARs, particularly at $\mathrm{CH}$ boundaries (e.g., Baker et al. 2007). Open flux is transferred to the edge of the AR postreconnection (consistent with the outflows at these locations). Evidence of interchange reconnection contributing to AR SW has been found at high-altitude magnetic nulls above ARs (Del Zanna et al. 2011) and at QSLs in close proximity to PFSS open magnetic field (van Driel-Gesztelyi et al. 2012).

Second, Culhane et al. (2014) and Mandrini et al. (2014) found evidence of AR plasma escaping to the heliosphere through a multistepped reconnection process, involving at least one instance of closed-closed field line reconnection, followed by reconnection at a high-altitude null point. These steps form a "chain" of reconnection.

Third, without the explicit requirement of reconnection, plasma can escape into the SW through the expansion of AR loops. This expansion has been observed to continually occur (Uchida et al. 1992) and has been observed at distances of $>12 R_{\odot}$ (Morgan et al. 2013). At these distances the loops themselves should be considered a part of the SW.

\subsection{Case Study of SW Associated with AR 12532}

From the above, it is clear that the mechanisms through which an AR may contribute SW to the heliosphere are varied and often complex. Case studies of AR SW are thus crucial in exploring these mechanisms. This paper presents a case study of two SW periods, associated with the same low-latitude $\mathrm{CH}$, separated by one solar rotation. The first features a $\mathrm{CH}$ with a simple $\mathrm{CH}$-quiet Sun ( $\mathrm{CH}-\mathrm{QS})$ trailing boundary, while the second features the same $\mathrm{CH}$, but now with a newly emerged AR (AR 12532) at its eastern edge, creating a $\mathrm{CH}-\mathrm{AR}$ boundary. This configuration presents an opportunity to contrast the differences in SW associated with the trailing $\mathrm{CH}-\mathrm{QS}$ and $\mathrm{CH}-\mathrm{AR}$ boundaries, which we capitalize on in this study. We thus aim to isolate the effects of an AR on the SW escaping a $\mathrm{CH}$ and so draw robust conclusions on the processes responsible for SW contribution from ARs.

The structure of this paper is as follows. In Section 2 we describe the data used in the study, how observations are selected, the backmapping procedure, and the observational signatures we expect for AR SW. Section 3 describes the key results derived from the observations. In Section 4 we discuss the observations both before and after AR 12532 emerges, and identify and explain the changes to the SW that arise as a result. We draw conclusions in Section 5.

\section{Data and Methodology}

\subsection{In situ and Remote Sensing Data}

The two Carrington rotations studied in this paper are CR 2175 and CR 2176, which we shall refer to as "R1" and "R2," respectively. For both R1 and R2 the "regions of interest" for all remote sensing observations are a persistent $\mathrm{CH}$ and surrounding structures.

SW data are obtained from the ACE (Stone et al. 1998) and Wind (Ogilvie \& Desch 1997) spacecraft at L1. SW bulk speed data $\left(v_{\mathrm{sw}}\right)$ are obtained at 1-minute resolution from $A C E-$ SWEPAM (McComas et al. 1998). Carbon charge state ratio, $\mathrm{C}^{6+} / \mathrm{C}^{5+}$, and iron abundance measured relative to oxygen, $\mathrm{Fe} / \mathrm{O}$, data are from ACE-SWICS (Gloeckler et al. 1992) available on a $2 \mathrm{hr}$ time resolution.

Vector magnetic field data, $B$, are obtained from the $A C E$ Magnetic Fields Experiment (Smith et al. 1998) at 4-minute resolution. To compare with composition data, we smooth the magnetic field components in time to a $2 \mathrm{hr}$ resolution. We label the field as sunward or antisunward polarity, following authors such as Owens et al. (2013) in defining the field that is within $\pm 90^{\circ}$ of the radial outward direction as antisunward (positive), while others are sunward (negative).

Suprathermal electron flux data at L1 are obtained from Wind-3DP (Lin et al. 1995). We consider the electrons in the $\sim 427 \mathrm{eV}$ energy bin, which clearly shows the suprathermal, beamed, SW electron population, known as the strahl, imposed over the quasi-isotropic halo (more information on these populations can be found in, e.g., Pierrard et al. 2001).

Candidate SW source regions are studied using remote sensing observations from the Solar Dynamics Observatory (SDO; Pesnell 2015) and Hinode (Kosugi et al. 2007) spacecraft. Fulldisk coronal images are obtained in the $193 \AA$ band from the Atmospheric Imaging Assembly (AIA) on SDO (Lemen et al. 2011). Line-of-sight (LOS) photospheric magnetogram observations are obtained from SDO's Helioseismic and Magnetic Imager (HMI; Scherrer et al. 2012).

Coronal EUV intensity and plasma parameters are derived from Hinode/EIS observations. In particular, these data are from measurements made at 10:06 UT on 2016 March 25 and at 05:45 and 10:00 UT on 2016 April 21, obtained with the $2^{\prime \prime}$ slit in scanning mode. For the observations taken at 10:06 UT on 2016 March 25 and at 05:45 UT on 2016 April 21, the large field of view (FOV) of $492^{\prime \prime} \times 512^{\prime \prime}$ is constructed by taking $60 \mathrm{~s}$ exposures at each of the 123 pointing positions with a scan step size of $4^{\prime \prime}$. For the observation at 10:00 UT on 2016 April 21, the FOV of $240^{\prime \prime} \times 512^{\prime \prime}$ is constructed from 120 positions and a step size of $2^{\prime \prime}$. Data processing and calibration are carried out using standard EIS SolarSoft ${ }^{\dagger}$ routines. Raw data have been corrected for hot, warm, and dusty pixels, cosmic rays, and dark current. Instrumental effects such as CCD detector offset, slit tilt, and orbital variation are also corrected. The calibrated spectra are fitted with a single Gaussian function except in the cases where there are known

\footnotetext{
7 Available at http://www.lmsal.com/solarsoft.
} 
blends, e.g., the Fe XII $\lambda 195.12$ emission line, which we use to create the intensity, Doppler, and nonthermal velocity $\left(v_{n t}\right)$ maps. An intensity thresholding technique (Krista \& Gallagher 2009 ) is applied, in which the local minimum value after the $\mathrm{CH}$ intensity peak for each raster is the cutoff level below which the pixels are masked (see Baker et al. 2018, for more details). Reference wavelengths are taken from the average value of relatively quiescent regions of each raster away from the $\mathrm{CH}$ and $\mathrm{AR}$ and used in the calculation of Doppler velocity.

We make FIP bias measurements of upflowing plasma within specific regions chosen for study. For each region, we select a smaller box containing only upflowing plasma and derive the mean FIP bias within it, following Brooks \& Warren (2011). The mean FIP bias value of each box is determined by averaging profiles for all spectral lines across all of the pixels within the box and then fitting the summed profiles with single or multiple Gaussian functions, depending on whether the lines contain blends. The spectral lines used include the Fe XIII 202.02 $\AA$ and $203.83 \AA$ density-sensitive line pair, the high-FIP S X $264.22 \AA$ and the low-FIP Si X $258.38 \AA$ lines, and a series of strong spectral lines from consecutive ionization stages of $\mathrm{Fe}$ VIII-XVI. We use the CHIANTI Atomic Database, version 8.0 (Dere et al. 1997; Del Zanna et al. 2015), to calculate the contribution functions for all spectral lines, assuming the $\mathrm{Fe}$ XIII-measured densities and adopting the photospheric abundances of Grevesse et al. (2007). Emission measure distributions are computed from the Fe VIII-XVI lines using the Markov Chain Monte Carlo algorithm available in the PINTofALE software package (Kashyap \& Drake 2000) and then convolved with the contribution functions and fitted to the observed intensities of the spectral lines of the low-FIP element $\mathrm{Fe}$. The emission measure is scaled to reproduce the Si X line intensity, as it is also a low-FIP element. FIP bias, then, is the ratio of the predicted to observed intensity for the high-FIP element $S \mathrm{X}$ line. This method accounts for residual temperature and density effects on the FIP Si X-S X line ratio and is used in, e.g., Brooks \& Warren (2011), Baker et al. (2013), and Brooks et al. (2015).

\subsection{Mapping of Solar Wind Streams}

In situ and remote sensing observations are linked using a standard two-step ballistic backmapping approach (as used by, e.g., Neugebauer et al. 1998; Ko et al. 2014; Fu et al. 2015; Fazakerley et al. 2016; Heidrich-Meisner et al. 2016). The first step calculates the Sun to spacecraft travel time for a constant, radial SW, moving at the speed measured at L1, as in Nolte \& Roelof (1973). This time is used to map to a location on the source surface of a PFSS coronal magnetic field model (Schatten et al. 1969). We obtain and analyze PFSS models using the "pfss" software included with the IDL SolarSoft package. We choose a source surface radius of $2.5 R_{\odot}$, and the pfss software combines concurrent magnetogram observations and a flux transport model to set the lower boundary conditions (for details on this, see Schrijver \& DeRosa 2003). In this way we obtain a mapping from source surface to photosphere via open field lines, identifying a source location, or "sourcepoint," at $1 R_{\odot}$ for each data point measured on a $2 \mathrm{hr}$ cadence by $A C E-$ SWICS at L1. The PFSS model also provides polarity information for the mapped source region, to later be compared to the polarity observed in the in situ interplanetary magnetic field (IMF) data.

\subsection{Key Observable Signatures for AR-associated SW}

We now describe observable signatures that we will use in the coming sections to identify SW of AR origins and possible mechanisms to produce it. Doppler observations of upflowing plasma, when located near open flux footpoints, suggest that plasma may escape into the heliosphere. This signature should manifest toward the edge of the source AR in the simple cases of interchange reconnection and loop expansion. Chained reconnection, in which multiple steps are needed for plasma to escape into the heliosphere, can be associated with upflow signatures, but the magnetic flux cannot be open to the heliosphere (as in Mandrini et al. 2014, where the AR was confined below the streamer belt). Enhancements in $v_{\text {nt }}$ have been found to correlate with upflows near ARs (Doschek et al. 2008). In addition to waves, turbulence, and unresolved bulk plasma motions, $v_{n t}$ enhancements have been linked to plasma motion associated with reconnection events (Parker 1988; Harra et al. 2001). Given that these possible explanations of $v_{\mathrm{nt}}$ enhancement are numerous and not fully understood, it is here viewed as supporting, and not primary, evidence of reconnection.

In situ compositional observations may give evidence of source region reconnection. The release of plasma from different-sized loops may manifest as variation in in situ charge states (as was argued by Fazakerley et al. 2016) since loop temperature correlates with loop length (Rosner et al. 1978). Such variation might also occur through opening similar loops at different heights, due to nonuniformity of temperature along a coronal loop (e.g., Huang et al. 2012). Similar variability in elemental abundance is expected when loops containing variously FIP-biased plasma (as is common for ARs; Baker et al. 2013) are opened.

Source mechanisms can also be inferred from the topology of the IMF, which is probed using the orientation of the SW strahl. Closed magnetic loops in the SW, consistent with AR loop expansion, can be identified from bidirectional or "counterstreaming" strahl (Montgomery et al. 1974; Pilipp et al. 1987). Counterstreaming strahl is also traditionally associated with ICMEs (Gosling et al. 1987); however, AR loops should be separable from ICMEs through observations of lower charge states, due to the lack of flaring (Gopalswamy et al. 2013).

Inverted (or "kinked") magnetic field, as identified by sunward-flowing strahl, can indicate interchange reconnection near the Sun (e.g., Crooker et al. 2004; Baker et al. 2009; Owens et al. 2013, 2018). Kinks may form from the opening of larger coronal loops (Owens et al. 2013) and then propagate out into the heliosphere, straightening out at around the local Alfvén speed (Gosling et al. 2005). Alternatively, kinks that are supported by velocity shear along magnetic flux tubes (which may naturally result from the opening of closed loops; see Figure 3 of Owens et al. 2018) can exist in the heliosphere for as long as the shear persists.

We note some further details on inverted IMF. First, kinks in the field created by reconnection may initially invert but partially straighten before being observed as only a deflection from the Parker spiral direction (see Lockwood et al. 2019). Second, reconnection inferred from these in situ magnetic field signatures need not be associated with a corresponding enhancement in $v_{\mathrm{nt}}$ in solar imagery. Finally, waves and turbulent fluctuations over a range of scales may also produce deflections in the IMF (Bruno \& Carbone 2013). While these 

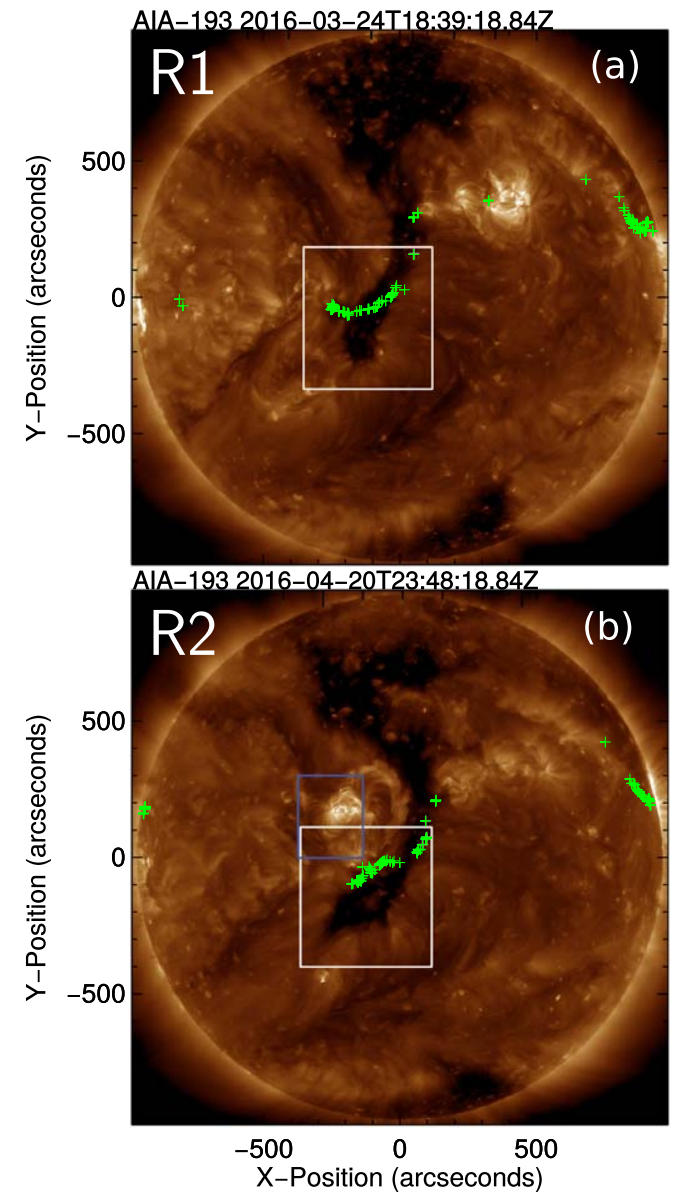

Figure 1. AIA-193 $\AA$ images of the Sun during (a) R1 and (b) R2. The $\mathrm{CH}$ is positioned at approximately disk center in both cases. Mapped SW sourcepoints (green plus signs, $2 \mathrm{hr}$ cadence) show that wind measured at $A C E$ maps to this $\mathrm{CH}$ and its trailing boundary for both $\mathrm{R} 1$ and $\mathrm{R} 2$. In $\mathrm{R} 1$, the $\mathrm{CH}$ is surrounded by QS (other than the AR to the northwest of the $\mathrm{CH}$ ). In $\mathrm{R} 2$, an AR has emerged at the eastward edge of the $\mathrm{CH}$, and the $\mathrm{AR}$ to the northwest has decayed. The morphology of the $\mathrm{CH}$ and sourcepoint locations are similar between R1 and R2 despite the emergence of the AR. The white and blue boxes show the FOV of EIS observations described later in the paper.

deflections could produce inversions of the IMF, we do not expect these inversions to necessarily coincide with compositional structures, which cannot be changed by turbulence.

\section{Results}

\subsection{Overview of Observation Periods}

In studying regions of interest for R1 and R2, we choose observation times close to when that region is predicted to have produced $A C E$-directed $\mathrm{SW}$, based on the ballistic backmapping above, and also is near disk center. These two times are typically within a few days of one another. As an overview of the consecutive periods under study, Figure 1 shows fulldisk images of the source $\mathrm{CH}$ as imaged in $193 \AA$ with AIA. Figure 1(a) shows the source $\mathrm{CH}$ during R1, with mapped L1 SW sourcepoints plotted as green plus signs, and the FOV of Hinode-EIS observations of the same $\mathrm{CH}$ marked in white. The trailing eastward (left) boundary of the $\mathrm{CH}$ borders QS. There is an AR to the northwest of the CH. PFSS modeling predicts this AR to be a source of some open flux, although ballistic mapping results in only a single $2 \mathrm{hr}$ sourcepoint for L1 SW plasma within it.
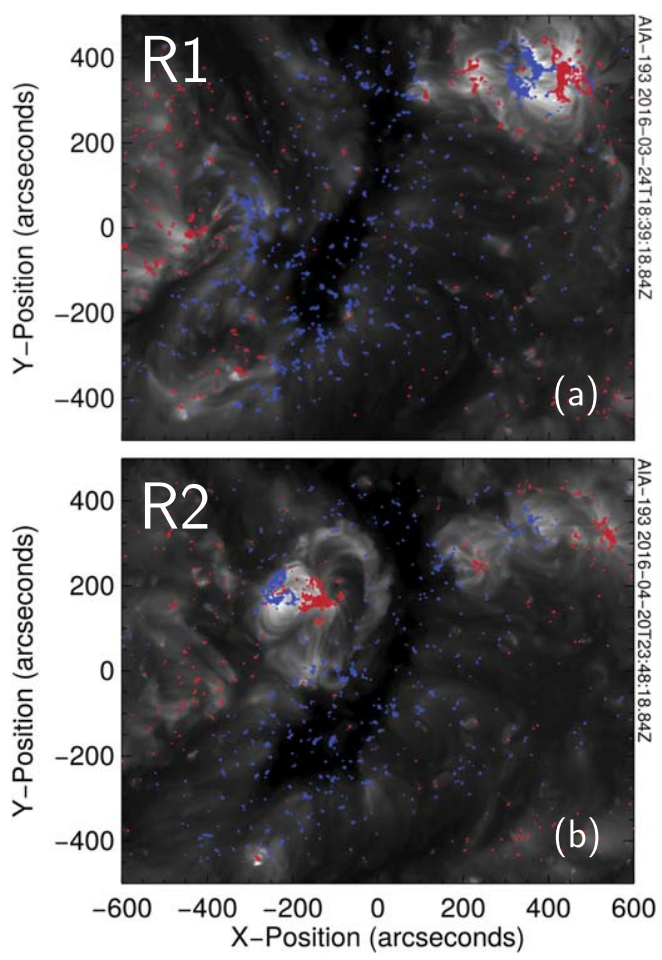

Figure 2. Cut-out AIA images of the Sun at the same time and channel as Figure 1, with HMI line-of-sight magnetogram contours overlaid on top. The AIA color table has been changed to gray scale to improve the visibility of the contours. The R1 and R2 HMI observations are from 2016 March 24 18:37:59 UTC and 2016 April 20 23:46:18 UTC respectively. The contour value is \pm 200 G. Blue contours indicate a positive (antisunward) LOS component of flux, while red is negative. The AR to the east of the $\mathrm{CH}$ in $\mathrm{R} 2$ is revealed to be dipolar in this image. In $\mathrm{R} 2$ the $\mathrm{CH}$ and the trailing boundary region that is crossed by SW sourcepoints in Figure 1 are both broadly unipolar and positive. The negative-polarity footpoint of the AR is found to be the footpoint closest to the positive-polarity $\mathrm{CH}$.

Figure 1(b) shows the same $\mathrm{CH}$ during R2. Again, $2 \mathrm{hr}$ mapped SW sourcepoints are plotted as green plus signs. White and blue boxes now show boundaries of EIS observations made of the $\mathrm{CH}$ and $\mathrm{AR}$, respectively. The AR located to the northwest of the $\mathrm{CH}$ during $\mathrm{R} 1$ has decayed substantially. However, on the eastern (left) side of the $\mathrm{CH}$ a new AR, AR 12532, has emerged since the previous rotation. AR 12532 lies to the north of the mapped SW sourcepoints at the trailing $\mathrm{CH}$ boundary. For R1 and R2, SW sourcepoints that appear to map to either limb correspond to streams before and after the $\mathrm{CH}$ streams of interest for these rotations.

Comparing the $\mathrm{CH}$ structure between $\mathrm{R} 1$ and $\mathrm{R} 2$, we note that its general morphology is qualitatively similar. The mapping of sourcepoint locations is also very similar between $\mathrm{R} 1$ and R2, despite the emergence of AR 12532. The preservation of $\mathrm{CH}$ morphology and mapping location suggests that any changes to the nature of the SW between R1 and R2 should be primarily a result of the emergence of the AR. Over the course of each observational period there is also little activity and flaring (a single B-class flare occurs for AR 12532 during R2), suggesting that the SW from these regions is produced under quasi-steady conditions, rather than through large sporadic events.

In Figure 2 we show magnetic flux data from HMI LOS magnetograms overlaid on the AIA imagery. The images are subfields of those in Figure 1, centered around the source CHs. The HMI contours are from observations within $12 \mathrm{~s}$ of the 

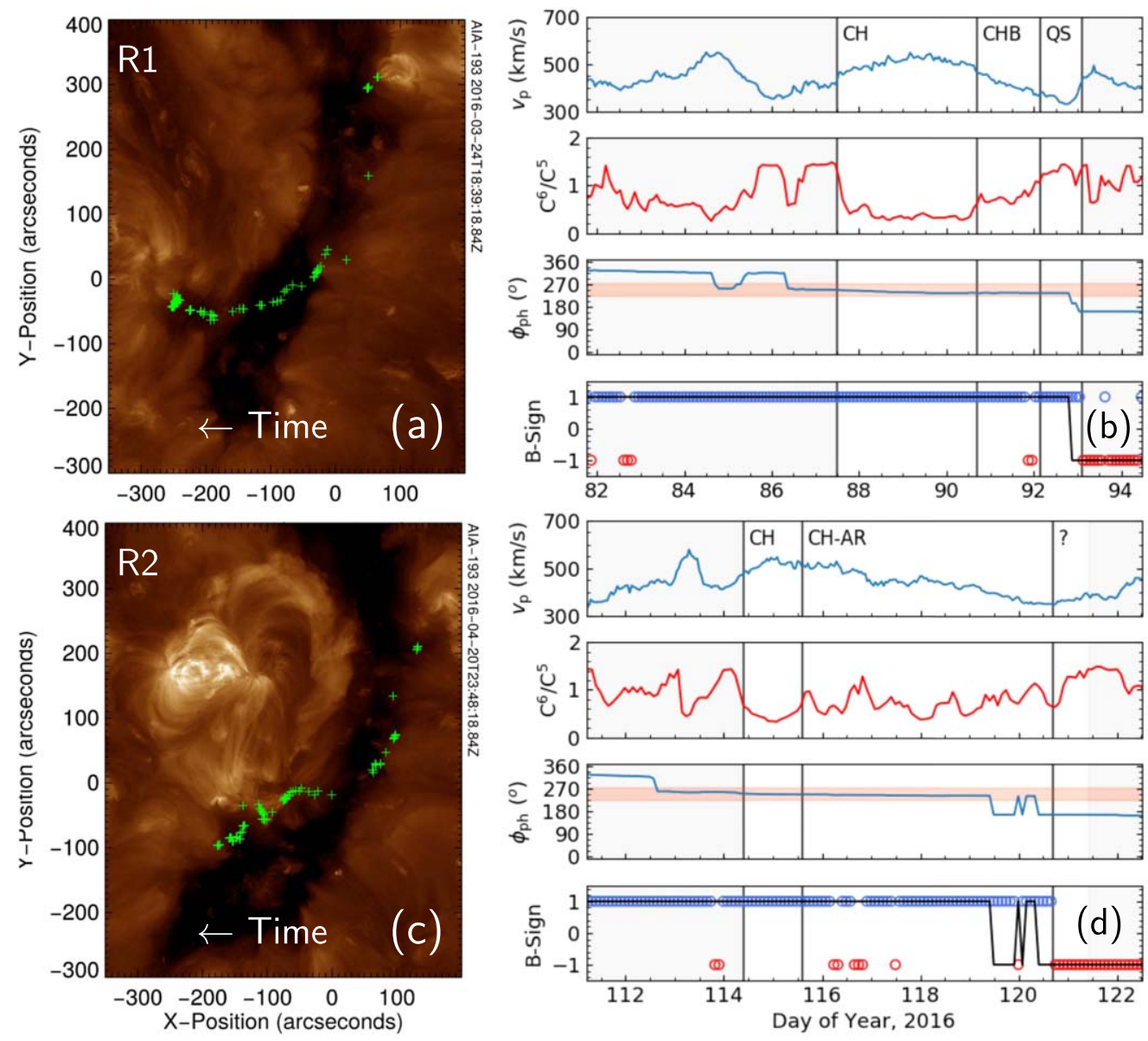

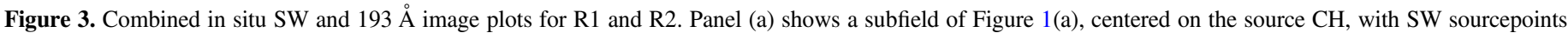

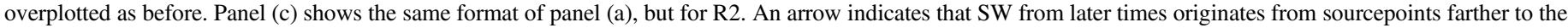

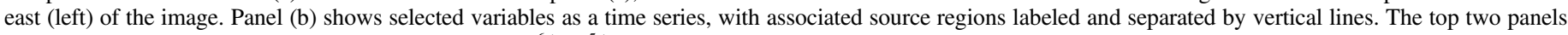

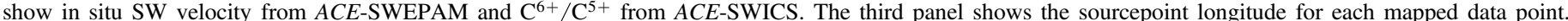

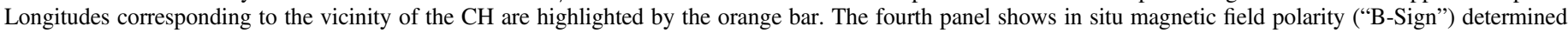

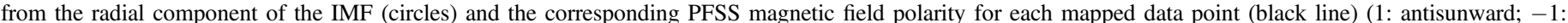

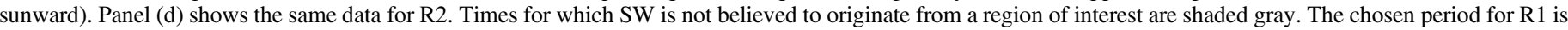

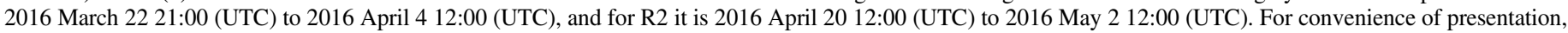
we plot time in day of year (DoY) format, in the range of $\sim 81.5-94.5,2016$ for R1 and $\sim 110.5-122.5,2016$ for R2.

AIA images. The polarities of the $\mathrm{CH}$, across the $\mathrm{AR}$, and at the $\mathrm{CH}$ boundary are all steady over the periods of interest for this study. We see for R1 in Figure 2(a) that the source $\mathrm{CH}$ is predominantly unipolar, in this case with positive (i.e., outward) field. The field inverts some $100^{\prime \prime}-300^{\prime \prime}$ due east from the $\mathrm{CH}$ boundary.

Figure 2(b) shows that for R2 the unipolarity of the $\mathrm{CH}$ is preserved, as is the predominantly positive polarity of the surrounding boundary. To the east of the $\mathrm{CH}$, QS regions change polarity $\sim 300^{\prime \prime}$ from the $\mathrm{CH}$ edge. At the footpoints of the bright loops of AR 12532, we find a dipolar configuration. This is oriented such that the negative polarity is adjacent to the $\mathrm{CH}$. Dimmer "AR-connected" loops extend from the $\mathrm{CH}$ boundary, also joining to the negative polarity footpoint of the AR. Some of these loops are rooted in the $\mathrm{CH}$ boundary region, where SW sourcepoints are predicted to be located (Figure 1).

\subsection{Linked Observations}

To identify probable source regions/in situ periods of interest for R1 and R2, we consider in more detail the mapped SW sourcepoints. Figures 3(a) and (c) show the $\mathrm{CH}$ and other features during R1 and R2 more closely. The images are AIA subfields at the same helioprojective coordinates, one solar rotation apart.

Figures 3(b) and (d) plot the associated in situ data for the observations shown in Figures 3(a) and (c), respectively. The time ranges we choose for in situ periods of interest are those that definitively map to the source regions of interest. We also include surrounding periods lasting 2-6 days to give context. The data are plotted against measurement time at L1. The SW associated with the $\mathrm{CH}$ and $\mathrm{CH}$ boundary through the mapping for $\mathrm{R} 1$ is estimated to be released over DoY $\sim 83-87$ (2016 March 23-27). The $\mathrm{CH}$ and AR-associated wind for $\mathrm{R} 2$ are estimated to be released over DoY 110-116 2016 (2016 April 19-25).

The top two panels of Figures 3(b) and (d) show ACE bulk SW speed, $v_{\mathrm{p}}$, and $\mathrm{C}^{6+} / \mathrm{C}^{5+}$ observations. The mapped photospheric Carrington longitudes, $\phi_{\text {phot }}$, in the third panels provide an indication of when sourcepoints change location gradually or rapidly. The longitude range that corresponds to the vicinity of the $\mathrm{CH}$ is highlighted in orange. The bottom panels show IMF polarity (red and blue circles; 1 is antisunward, -1 is sunward) as calculated from the radial 
component of the IMF observed in situ. The polarity of the PFSS magnetic field at the corresponding sourcepoints is also shown (solid black line).

Due to the numerous limitations in the ballistic mapping procedure (Nolte \& Roelof 1973; Neugebauer et al. 1998; Riley \& Lionello 2011) and PFSS modeling (e.g., Schatten et al. 1969; Riley et al. 2006), we do not expect precise agreement between features in SW streams and the mapped source locations. We thus use evidence from SW properties to identify the relevant streams for each rotation. Both $\mathrm{R} 1$ and $\mathrm{R} 2$ feature long-lived ( $>1$ day) streams of $v_{\mathrm{p}}>500 \mathrm{~km} \mathrm{~s}^{-1}$ and reduced $\mathrm{C}^{6+} / \mathrm{C}^{5+}(\lesssim 0.6)$, which are thus likely to be associated with the $\mathrm{CH}$ and generally map well to within its vicinity based on $\phi_{\text {phot }}$. Both R1 and R2 feature shorter-lived fast streams preceding them, which also map to the vicinity of the $\mathrm{CH}$. Identification of the onset time for the $\mathrm{CH}$ stream for each case is not paramount, as the primary period of interest is the trailing portion (as this corresponds to the eastward $\mathrm{CH}$ boundary). We thus label the onset of the long-lived fast stream with depleted $\mathrm{C}^{6+} / \mathrm{C}^{5+}$ as the start of the $\mathrm{CH}$ streams (vertical black line in the figure) for both R1 and R2.

For $\mathrm{R} 1$, following the onset of the $\mathrm{CH}$ stream, $v_{\mathrm{p}}$ and $\mathrm{C}^{6+} / \mathrm{C}^{5+}$ persist at similar levels for $\sim 3$ days, before $v_{\mathrm{p}}$ begins a gradual decrease. Here $\mathrm{C}^{6+} / \mathrm{C}^{5+}$ rises more rapidly to a value intermediate between $\mathrm{CH}$ and pre- $\mathrm{CH}$ levels. This rise is characteristic of a $\mathrm{CH}$ boundary $/ \mathrm{CH}$ boundary layer $(\mathrm{CHB})$ as discussed by, e.g., McComas (2003) and Schwadron et al. (2005), and so we label this period as such. Shortly thereafter, $\mathrm{C}^{6+} / \mathrm{C}^{5+}$ begins to climb again before settling on pre- $\mathrm{CH}$ levels, while $v_{\mathrm{p}}$ continues to fall. We label this period as "QS" simply to separate it from the preceding region, from which it is compositionally distinct. The HMI magnetogram (Figure 2(a)) indicates that only wind of antisunward magnetic polarity should originate from the $\mathrm{CH}$ and $\mathrm{CH}-\mathrm{QS}$ boundary. We thus end the QS period of interest at the point where the in situ IMF polarity flips. The in situ magnetic field polarity is predominantly antisunward for these three periods, and for the majority of the time the mapped PFSS polarity is in agreement.

For $\mathrm{R} 2, v_{\mathrm{p}}$ and $\mathrm{C}^{6+} / \mathrm{C}^{5+}$ persist at $\mathrm{CH}$ levels for only $\sim 1$ day following the onset of the $\mathrm{CH}$ stream. Following this, $\mathrm{C}^{6+} / \mathrm{C}^{5+}$ proceeds to fluctuate for around 5 days. Over this time $v_{\mathrm{p}}$ decreases, but not in a steady fashion as in $\mathrm{R} 1$. We mark this entire region as "CH-AR," as it is possible that different streams within this region may originate from the $\mathrm{CH}$, $\mathrm{AR}$, or the AR-connected boundary, as described above.

There is poor agreement toward the latter part of the $\mathrm{CH}-\mathrm{AR}$ period between PFSS and in situ polarity; PFSS polarity flips rapidly, while the in situ polarity remains predominantly antisunward, before switching to sunward. We attribute this to mapping errors, possibly resulting from so-called "dwells" (Riley \& Lionello 2011) arising during the mapping of this rarefaction region $\mathrm{SW}$. As in $\mathrm{R} 1$, we are confident that the entire +1 polarity part of the $\mathrm{CH}-\mathrm{AR}$ period is in fact from the $\mathrm{CH}-\mathrm{AR}$ region. The -1 polarity period should primarily originate from structures across the HCS from the regions of interest, which are predominantly positive polarity. However, this period might also include plasma from the negative part of the AR proper (should plasma from this region be able to escape into the heliosphere). We thus mark the end of the $\mathrm{CH}-$ AR period at the location where IMF polarity inverts, but we also mark the stream that immediately follows this inversion as ambiguous with "?."

\subsection{Physical Properties of Source Regions}

In Figures 4(a)-(c), we show observations of parameters derived from ultraviolet spectral images from Hinode-EIS centered on the $\mathrm{CH}$ during R1. Panel (a) shows the Fe XII intensity map, which matches the $193 \AA$ imagery shown in Figure 1, albeit with lower spatial resolution. The Doppler velocity map in panel (b) shows that the $\mathrm{CH}$ contains predominantly upflowing plasma, on the order of around $10-20 \mathrm{~km} \mathrm{~s}^{-1}$. At the eastward $\mathrm{CH}$ boundary, where sourcepoints approach QS, there is a mixture of strong and weak upflow and downflow regions. We highlight two regions (Boxes 1 and 2) that feature enhancement in upflow near this location. Panel (c) maps $v_{\mathrm{nt}}$, derived from the width of the $\mathrm{Fe}$ XII line. We note that $v_{\text {nt }}$ of up to $\sim 40-60 \mathrm{~km} \mathrm{~s}^{-1}$ can be found in the $\mathrm{CH}$ (although the data are approaching the noise threshold, due to low counts in Fe XII, as seen in panel (a)). Surrounding the $\mathrm{CH}$, background $v_{\mathrm{nt}}$ is around $15-25 \mathrm{~km} \mathrm{~s}^{-1}$. Boxes 1 and 2 both feature regions of enhanced $v_{\mathrm{nt}}$, colocated with upflows. We apply the FIP bias measurement procedure described in Section 2.1 to Boxes 1 and 2 and record the corresponding averaged FIP bias values in Table 1. Upflowing material in both boxes displays enhanced FIP bias, with Box 2 being the greater of the two.

EIS observations of the source $\mathrm{CH}$ during $\mathrm{R} 2$ are shown in Figures 4(d)-(f). Panel (d) shows the Fe XII intensity. The Doppler velocity map in panel (e) shows that the $\mathrm{CH}$ still contains upflowing plasma, with velocities on the same order as during $\mathrm{R} 1$. Box 3, along the $\mathrm{CH}$ boundary and sourcepoint path, features strong upflow, while Box 4 features a weaker upflow but is located farther from the $\mathrm{CH}$ boundary and near the bright AR-connected loop footpoints. Again, $v_{\mathrm{nt}}$ observations in panel (f) are largely noise in the $\mathrm{CH}$ and inner portion of the boundary. However, this appears to be where most enhanced $v_{\mathrm{nt}}$ is found. Further to the north, in the ARconnected boundary, very low $v_{\mathrm{nt}}$ values are observed. Box 3 contains localized strong $v_{\mathrm{nt}}$ enhancement, while Box 4 shows low $v_{\text {nt }}$. Mean FIP biases in Boxes 3 and 4 are again recorded in Table 1. Box 3 FIP bias is clearly enhanced (similar to Box 1 for R1), while Box 4 FIP bias is only weakly enhanced (although still $>1$ ).

Figures 4(g)-(i) show the same observations for AR 12532 as are shown for the $\mathrm{CH}$ in Figures 4(a)-(c). The FOV of these observations is indicated by the blue box in Figure 1(b). The polarity of the field is now important, as the AR is dipolar. We draw comparison with HMI contours in Figure 2 to determine the polarity of the photosphere near a given feature. In panel $(\mathrm{g})$ the core of the AR is visible in Fe XII in the right half of the image. The negative-polarity region of the AR core is cut off at the edge of the map. In panel (h) the Doppler velocity shows a region with upflows of $\sim 20 \mathrm{~km} \mathrm{~s}^{-1}$ to the east, and particularly northeast of the AR core, highlighted at Box 5. We also highlight Box 6, a smaller upflowing region lying close to the AR core. Boxes 5 and 6 both outline primarily positive-polarity regions. Panel (i) shows that $v_{\mathrm{nt}}$ is uniform for much of the FOV, at around $10-20 \mathrm{~km} \mathrm{~s}^{-1}$. One exception is within the dimmer loops at the core of the AR, where $v_{\mathrm{nt}}$ approaches zero. Another notable exception is in the strong upflow region in Box 5. Table 1 shows mean FIP bias within Boxes 5 and 6 . Both are moderately enhanced, but they are weaker than the enhancements found for Figures 4(a)-(c).

We apply the same PFSS model as is used in the backmapping process to show locations of open flux footpoints 

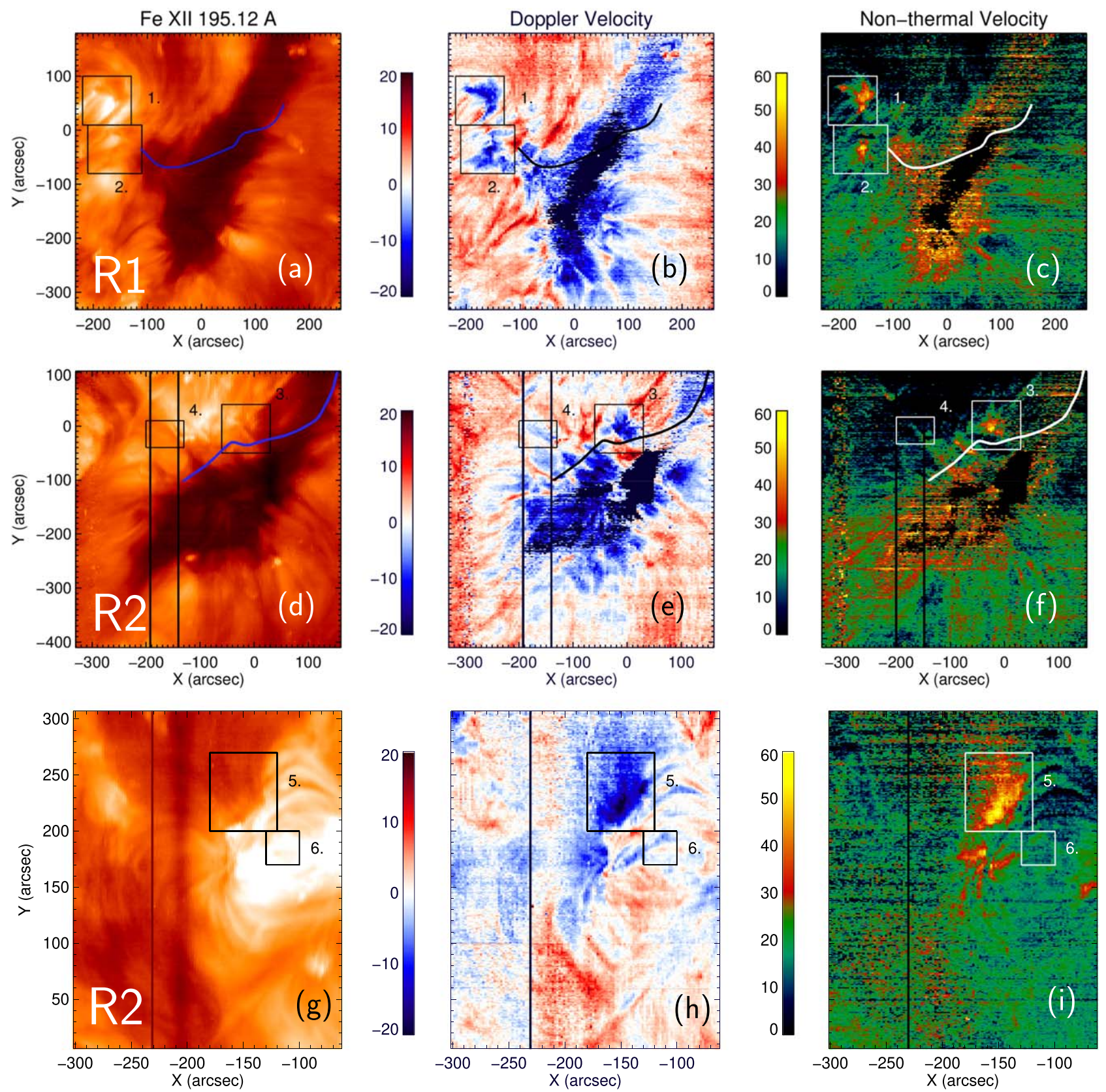

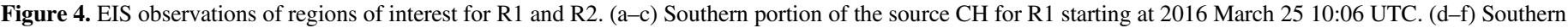

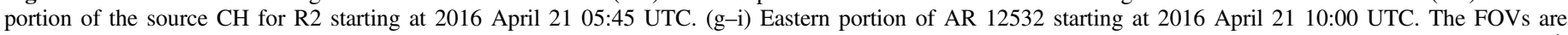

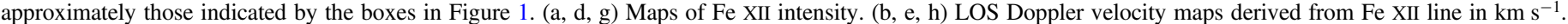

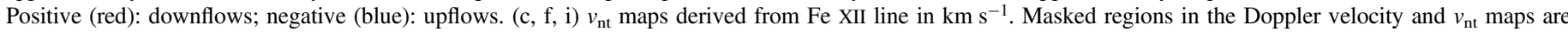

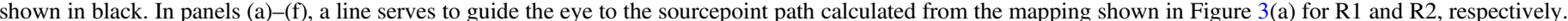

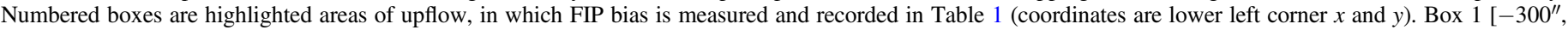
$\left.300^{\prime \prime}\right]$, Box $2\left[-300^{\prime \prime},-300^{\prime \prime}\right]$, Box $3\left[-300^{\prime \prime},-300^{\prime \prime}\right]$, Box $4\left[-300^{\prime \prime},-300^{\prime \prime}\right]$, Box $5\left[-300^{\prime \prime}, 300^{\prime \prime}\right]$, Box $6\left[-300^{\prime \prime}, 300^{\prime \prime}\right]$.

Table 1

Mean FIP Biases within Boxes Shown in Figure 4

\begin{tabular}{lccc}
\hline \hline EIS Observation & Region & Box & FIP Bias \\
\hline 2016 Mar 25-10:06 & CH-QS & 1 & 2.7 \\
& & 2 & 3.4 \\
2016 Apr 21-05:45 & CH-AR & 3 & 2.7 \\
& & 4 & 1.6 \\
2016 Apr 21-10:00 & AR & 5 & 2.3 \\
& & 6 & 2.2 \\
\hline
\end{tabular}

at $1 R_{\odot}$ for both R1 (panel (a)) and R2 (panel (b)) in Figure 5 . For $\mathrm{R} 1$, open flux is rooted in the EUV $\mathrm{CH}$ and the $\mathrm{CH}$ boundary, particularly to the east. The two locations of strong upflow, $v_{\mathrm{nt}}$, and enhanced FIP bias from Figures 4(a)-(c) (Boxes 1 and 2) map close to open flux rooted in the $\mathrm{CH}$ boundary in Figure 5(a).

Figure 5(b) shows corresponding open flux footpoints for both the $\mathrm{CH}$ and AR for R2. The coordinates on the image match as close as possible those in Figure 4(d), as the image time corresponds to the start time of the EIS rastering for this 

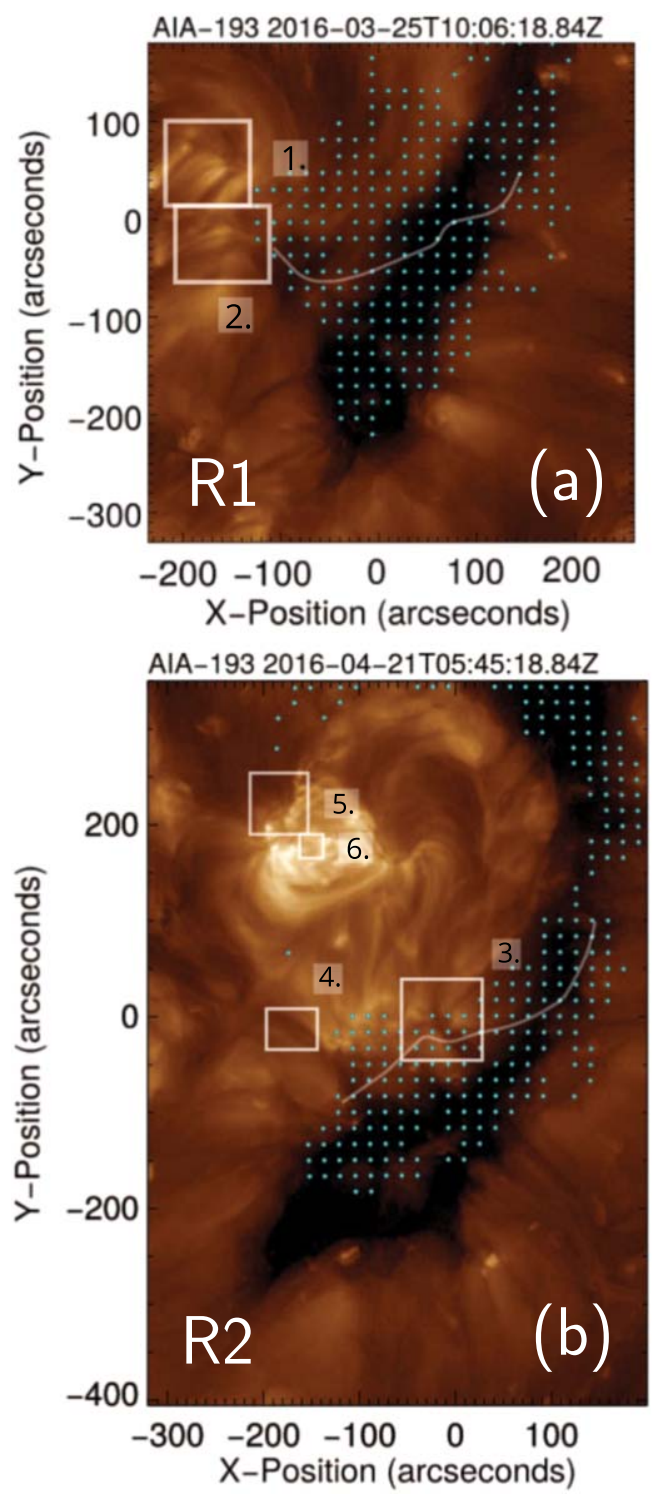

Figure 5. (a) Subregion of full-disk AIA $193 \AA$ images at time corresponding to EIS observation of the $\mathrm{CH}$ during $\mathrm{R} 1$ in Figure 4(a). The FOV is approximately the same as in Figure 4(a). Footpoints of open magnetic flux derived from the PFSS model are plotted in light blue based on a $1^{\circ}$-resolution grid at $1 R_{\odot}$. The line and boxes are the same as in Figure 4(a). (b) Same format as panel (a), but for the two EIS observations during R2 shown in Figures 4(d) and $(\mathrm{g})$. The FOV encloses both Figure 4(d) and (g) observations, and the image is taken at the time corresponding to the $\mathrm{CH}$ observations in Figure 4(d). The line and boxes are the same as in Figures 4(d) and (g). Boxes 5 and 6 have been shifted slightly to account for the different image times.

region. Open flux is found within the strong upflow/ $v_{\text {nt }} /$ fractionation region of Box 3 , and the AR-connected boundary in general. Open flux is also rooted close to, but not within, the weakly upflowing region in Box 4 and in the strong upflow region in Box 5. No such open flux is predicted to be rooted in the AR core.

To summarize these remote sensing results, we find that beyond structural differences and the presence of AR 12532 itself, the properties of the mapped source regions for R1 are broadly similar to those for $\mathrm{R} 2$. Around the $\mathrm{CH}$ and the QS and AR boundaries there are signatures of upflows, enhancement of $v_{\mathrm{nt}}$, and enhanced FIP bias. There are regions where all three of these are coincident and are adjacent to open flux footpoints predicted by the PFSS model. The FIP bias of the selected upflowing regions for $\mathrm{R} 1$ is greater than that for $\mathrm{R} 2$. The main structural difference in the $\mathrm{CH}$ boundary between $\mathrm{R} 1$ and $\mathrm{R} 2$ is that the former is adjacent to QS, while the latter is adjacent to brighter loops that are connected to the AR to the north.

\subsection{Detailed In Situ Observations}

Figures 6 and 7 plot all of the in situ data considered for both $\mathrm{R} 1$ and R2, respectively, in identical format. The first panels in each figure show fluxes of suprathermal electrons at $427 \mathrm{eV}$ as pitch-angle (PA) histograms. The PA bins approximately span from $0^{\circ}$ to $180^{\circ}$; Bin 1 is the bin looking nearest $0^{\circ}$, and Bin 8 is nearest $180^{\circ}$. We also plot the flux-weighted mean PA bin in white. The second panels of these figures show the same information as the fourth panel in Figures 3(b) and (d), without the PFSS polarity line, and instead with the strahl alignment derived from the mean PA bin.

The third panels plot $\phi_{\mathrm{r}}=180^{\circ}-\phi$, the difference between the azimuthal angle of $B$ in GSE coordinates $(\phi)$ and the radial antisunward direction $\left(180^{\circ}\right)$. Gray lines denote $\phi_{r}= \pm 90^{\circ}$, separating sunward and antisunward flux regions. The nominal Parker spiral direction relative to the radial direction, $\phi_{\mathrm{P}}$, is calculated using the expression given in Heidrich-Meisner et al. (2016). $\phi_{\mathrm{P}}$ is shown for both antisunward $\left(\sim 45^{\circ}\right)$ and sunward $\left(\sim-135^{\circ}\right)$ magnetic field directions as a purple line. We show the elevation angle, $\theta_{\mathrm{el}}$, of the IMF in the fourth panel; $0^{\circ}$ field is aligned with the ecliptic plane, while $>0^{\circ}\left(<0^{\circ}\right)$ field has a northward (southward) component. The fifth panel plots SW bulk velocity $v_{\mathrm{sw}}$. The sixth and seventh panels plot $\mathrm{C}^{6+} / \mathrm{C}^{5+}$ and $\mathrm{Fe} / \mathrm{O}$, respectively, each with error bars as provided with the $A C E$-SWICS data set. The associated FIP biases for the Fe/ $\mathrm{O}$ values are also shown. These are calculated by dividing the observation by the photospheric $\mathrm{Fe} / \mathrm{O}$ ratio; $\mathrm{Fe} / \mathrm{O}_{\text {phot }}=0.064$ from Asplund et al. (2009).

As shown in panel 1 of Figures 6 and 7, the strahl in both R1 and R2 is predominantly unidirectional. There are periods for both rotations in panel 2 during which the IMF switches polarity while the strahl alignment remains unchanged. The strahl in such cases is sunward, and we can infer that the field is locally inverted/ kinked (see Section 2.3). During the periods of interest, the number of IMF inversions as judged relative to the radial direction (crossings of the gray lines) is only 1 during $\mathrm{R} 1$, but 4 during R2.

We further quantify the number of instances where $\phi_{r}$ crosses, and subsequently returns from, the threshold of $\pm 45^{\circ}$ away from the nominal Parker direction, strongly deflecting without necessarily inverting fully. (For a typical Parker angle of $\sim 45^{\circ}$, these thresholds represent deflections to $0^{\circ}$ and $90^{\circ}$ relative to radial.) The number of instances for $\mathrm{R} 1$ is $n_{\mathrm{R} 1}=3$, while for R2 it is $n_{\mathrm{R} 2}=9$. Deflections in the IMF out of the ecliptic plane manifest as deviations of $\theta_{\mathrm{el}}$. Examining the $\mathrm{CH}-$ AR period following the $\mathrm{CH}$ stream, we also see numerous strong deviations in elevation.

Turning to composition, the ranges of measured $\mathrm{Fe} / \mathrm{O}$ and $\mathrm{C}^{6+} / \mathrm{C}^{5+}$ during both $\mathrm{R} 1$ and $\mathrm{R} 2$ span approximately the same values (in the range of $\sim 0.14-0.4$ ). We classify $\mathrm{SW}$ based on $\mathrm{Fe} / \mathrm{O}$ measurements from $\mathrm{R} 1$ and $\mathrm{R} 2$ directly, since here $\mathrm{Fe} / \mathrm{O}$ is particularly high in comparison to values reported in the literature (e.g., Kilpua et al. 2016; Fu et al. 2017). These values are nonetheless reliable, based on the SWICS quality flags. We interpret relatively high $\mathrm{Fe} / \mathrm{O}$ above some threshold as an indicator of origins outside of the $\mathrm{CH}$ for $\mathrm{R} 1$ and $\mathrm{R} 2$. Leveraging the initial in situ observations for $\mathrm{R} 1$, we define 


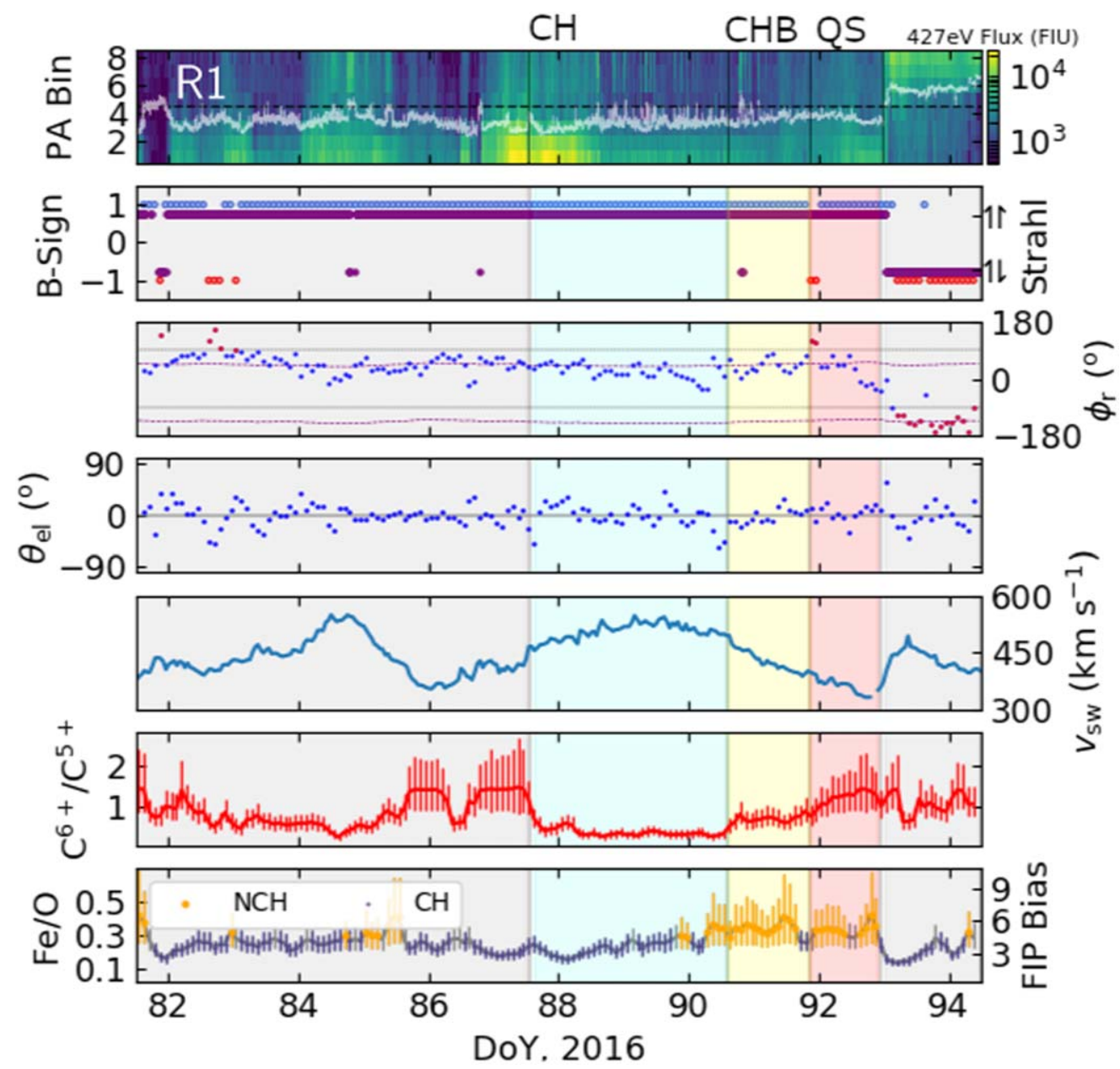

Figure 6. SW data from Wind and $A C E$ for the R1 in situ period. From top to bottom panels, these are as follows: (1) Wind-3DP suprathermal electron flux at $\sim 427 \mathrm{eV}$, by PA bin as a function of time, with units FlU $\left(=\mathrm{cm}^{-2} \mathrm{sr}^{-1} \mathrm{eV}^{-1} \mathrm{~s}^{-1}\right)$. The white line shows the flux-weighted mean PA. (2) IMF direction (1, blue, is antisunward; -1 , red, is sunward). Also shown in purple is the strahl alignment, derived from the mean PA bin. (3) $\phi_{r}$, the azimuthal angle between $B$ in GSE coordinates $(\phi)$ and the radial direction $\left(180^{\circ}\right.$ in GSE). Gray lines at $\pm 90^{\circ}$ show angles within which the magnetic field direction is considered to be antisunward relative to the radial direction. Dashed purple lines show the nominal Parker spiral angles. (4) Elevation angle, $\theta_{\mathrm{el}}$, of the IMF. (5) SW bulk velocity from $A C E$ SWEPAM. (6) $\mathrm{C}^{6+} / \mathrm{C}^{5+}$ from ACE-SWICS. (7) Fe/O also measured by SWICS, with the right side of the axis showing the inferred FIP bias: (Fe/O) $)_{\text {swICS }} /(\mathrm{Fe} /$ $\mathrm{O})_{\text {phot }}$. Points are color-shaded by apparent source as described in the text. $\mathrm{CH}, \mathrm{CHB}$, and QS sections of the plot are colored by $\mathrm{C}^{6+} / \mathrm{C}^{5+}$ value (details in the text). Regions that do not correspond to regions of interest are shaded gray.

thresholds based on R1 only. We split Fe/O into two rather than three classes, since the R1 CHB and QS regions, although clearly distinct in $\mathrm{C}^{6+} / \mathrm{C}^{5+}$, display similar $\mathrm{Fe} / \mathrm{O}$. We find the mean $\mathrm{Fe} / \mathrm{O}$ value in the $\mathrm{CH}$ period to be $\langle\mathrm{Fe} / \mathrm{O}\rangle_{\mathrm{CH}}=0.23$ and standard deviation $\sigma_{(\mathrm{CH})}=0.04$, while for the combined $\mathrm{CHB}$ and $\mathrm{QS}$ periods $\langle\mathrm{Fe} / \mathrm{O}\rangle_{\mathrm{NCH}}=0.33$ and $\sigma_{\mathrm{NCH}}=0.04$. In Figures 6 and 7 , points with $\mathrm{Fe} / \mathrm{O}<\langle\mathrm{Fe} / \mathrm{O}\rangle_{\mathrm{CH}}+\sigma_{\mathrm{CH}}$ are shown in purple and are characteristic of the $\mathrm{CH}$. Points with $\mathrm{Fe} / \mathrm{O}>\langle\mathrm{Fe} / \mathrm{O}\rangle_{\mathrm{NCH}}-\sigma_{\mathrm{NCH}}$ are marked orange and are characteristic of the R1 CHB or QS, which are "non$\mathrm{CH}$ " (NCH).

We perform a similar analysis for $\mathrm{C}^{6+} / \mathrm{C}^{5+}$ as for $\mathrm{Fe} / \mathrm{O}$. We shade the panels of Figure 6 by $\mathrm{CH}, \mathrm{CHB}$, and $\mathrm{QS}$ composition: blue for $\mathrm{CH}$ (low $\mathrm{C}^{6+} / \mathrm{C}^{5+}$ ), yellow for $\mathrm{CHB}$ (intermediate $\mathrm{C}^{6+} / \mathrm{C}^{5+}$ ), and red for QS (high $\mathrm{C}^{6+} / \mathrm{C}^{5+}$ ). For R2 in Figure 7 , both $\mathrm{C}^{6+} / \mathrm{C}^{5+}$ and $\mathrm{Fe} / \mathrm{O}$ compositional signatures change more rapidly than during $\mathrm{R} 1$. We draw boundaries between apparent structures in $\mathrm{C}^{6+} / \mathrm{C}^{5+}$ on timescales of $\sim 0.5-1$ days in Figure 7 . By comparing the mean $\mathrm{C}^{6+} / \mathrm{C}^{5+}$ for each of these structures in $\mathrm{R} 2$ to the mean $\mathrm{C}^{6+} / \mathrm{C}^{5+}$ for $\mathrm{CH}, \mathrm{CHB}$, and $\mathrm{QS}$ structures in $\mathrm{R} 1$, the $\mathrm{R} 2$ structures are colored by the same scheme as R1. We do not highlight the ambiguous negative-polarity region in R2.

In total, there are 10 separate $\mathrm{SW}$ stream structures identified in Figure 7 for R2, compared to the three in R1 upon which their identification is based. We label these structures sequentially S1-S10 (1-10 in the figure). We also label the ambiguous negative-polarity period as $\mathrm{S} 11$. The monotonically increasing charge state found in R1 is not preserved in R2. S1, the apparent $\mathrm{CH}$ stream for $\mathrm{R} 2$, persists for around 1 day, while the $\mathrm{CH}$ stream in $\mathrm{R} 1$ (which originates from the same $\mathrm{CH}$ ) persists for around 3 days. The strong deflections in $\phi_{r}$ occur on similar timescales to these structures we highlight in composition.

$\mathrm{Fe} / \mathrm{O}$ appears also to be split into small $\sim 0.5$ - to 1-day structures in the $\mathrm{CH}-\mathrm{AR}$ boundary of $\mathrm{R} 2$. These structures roughly align with $\mathrm{S} 1-\mathrm{S} 10$ derived from $\mathrm{C}^{6+} / \mathrm{C}^{5+}$. The three low- $\mathrm{C}^{6+} / \mathrm{C}^{5+}$ regions in $\mathrm{R} 2$ all correspond to low $\mathrm{Fe} / \mathrm{O}$. Of the five intermediate- $\mathrm{C}^{6+} / \mathrm{C}^{5+}$ regions, two (S2 and S5) correspond to low $\mathrm{Fe} / \mathrm{O}$ values and three (S3, S7, and S9) to high values. The two high $-\mathrm{C}^{6+} / \mathrm{C}^{5+}$ regions in $\mathrm{R} 2, \mathrm{~S} 4$ and $\mathrm{S} 10$, correspond to low and high $\mathrm{Fe} / \mathrm{O}$ values, respectively. 


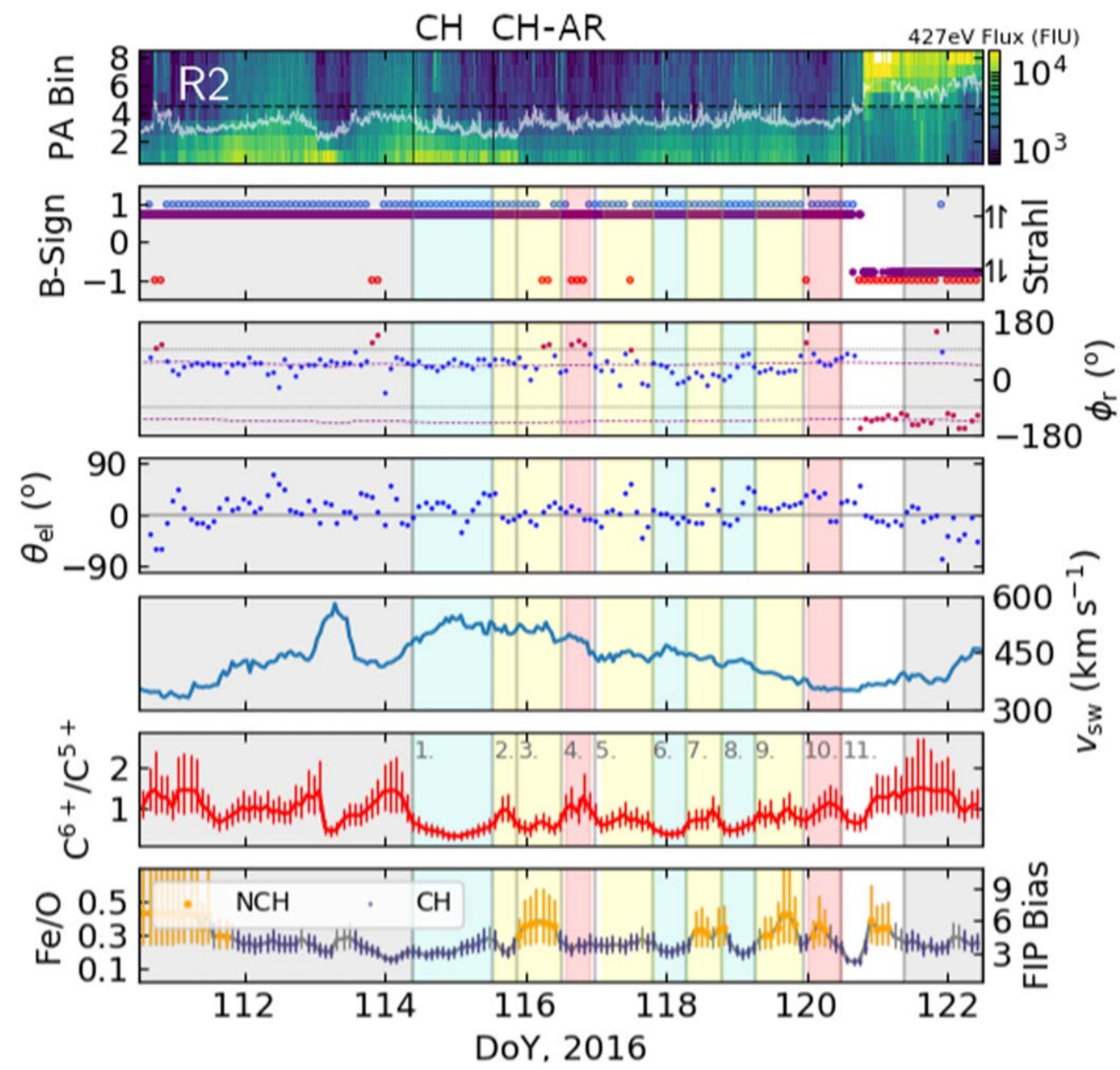

Figure 7. SW data from Wind and $A C E$ for the R2 in situ period. The panels are identical to Figure 6. Colored sections are numbered 1-11 and are referred to as S1$\mathrm{S} 11$ in the text. $\mathrm{CH}$ and $\mathrm{CH}-\mathrm{AR}$ regions as defined in Figure 3 are also labeled.

Combining the classifications by $\mathrm{C}^{6+} / \mathrm{C}^{5+}$ and $\mathrm{Fe} / \mathrm{O}$, we have found five of a possible six combinations of these classifications in R2.

To summarize, we collect the key comparative results from this section in Table 2. Absolute values of parameters such as $v_{\mathrm{sw}}, \mathrm{Fe} / \mathrm{O}$, and $\mathrm{C}^{6+} / \mathrm{C}^{5+}$ are very similar between $\mathrm{R} 1$ and $\mathrm{R} 2$. The main differences between the two periods are in fact in the stream structure, the variability of IMF orientation, and that R2 shows a greater variety of compositional signatures than R1.

\subsection{CH Boundary Comparison}

To contextualize the above in situ results, we compile a set of example trailing $\mathrm{CH}-\mathrm{QS}$ streams. We first select source $\mathrm{CHs}$. To ensure similarity to the $\mathrm{CH}$ during $\mathrm{R} 1$, we restrict to CHs that (1) occurred in the years 2016-2018, the declining/ minimum phase of solar cycle 24 (public $A C E$-SWICS data are available until 2018 June 8 UT); (2) have some portion that crosses disk center; (3) do not have an AR, as defined by both NOAA and SPoCA AR lists (Verbeeck et al. 2014) located on their eastern boundary; (4) are not concurrent with other $\mathrm{CHs}$ near disk center; and (5) are $>200^{\prime \prime}$ across at their broadest section when at central meridian. Following this process, 26 suitable $\mathrm{CHs}$ are identified.

Turning to in situ data from $A C E$, of the $26 \mathrm{CHs}$, we retain only those for which (1) a fast $\left(v \geqslant 450 \mathrm{~km} \mathrm{~s}^{-1}\right) \mathrm{SW}$ interval occurs at $A C E$ within $\sim 2-4$ days (the approximate SW travel
Table 2

Key Results for Section 3.4, for R1 and R2

\begin{tabular}{lcc}
\hline \hline Parameter & R1 & R2 \\
\hline Max. $v_{\text {sw }}\left(\mathrm{km} \mathrm{s}^{-1}\right)$ & 550 & 550 \\
Min. FIP bias & 1.7 & 1.8 \\
Max. FIP bias & 4.6 & 4.9 \\
Min. $\mathrm{C}^{6+} / \mathrm{C}^{5+}$ & 0.19 & 0.24 \\
Max. $\mathrm{C}^{6+} / \mathrm{C}^{5+}$ & 1.44 & 1.22 \\
Distinct structures & 3 & 10 \\
Fe $/ \mathrm{O}-\mathrm{C}^{6+} / \mathrm{C}^{5+}$ combinations & 3 & 5 \\
Instances of $\left|\Delta \phi_{p}\right|>45^{\circ}$ & 3 & 9 \\
Radial IMF inversions & 1 & 4 \\
CH stream duration (days) & 3 & 1 \\
\hline
\end{tabular}

Note. The rows from top to bottom are as follows: maximum SW bulk velocity, minimum FIP bias value, maximum FIP bias value, minimum $\mathrm{C}^{6+} / \mathrm{C}^{5+}$ value, maximum $\mathrm{C}^{6+} / \mathrm{C}^{5+}$ value, number of distinct structures identified by composition, number of combinations of $\mathrm{Fe} / \mathrm{O}$ and $\mathrm{C}^{6+} / \mathrm{C}^{5+}$ regimes, number of times $|\Delta \phi|$ exceeds and then falls below $45^{\circ}$, number of times the IMF inverts based on $\left|\Delta \phi_{\mathrm{P}}\right|$ and strahl, and the duration of the stream associated with the $\mathrm{CH}$.

time) of the $\mathrm{CH}$ appearance at disk center; (2) there is no evidence that multiple $\mathrm{CH}$ streams have merged (which would make it difficult to identify the trailing boundary); (3) an HCS crossing follows the $\mathrm{CH}$ stream and its trailing boundary, 
Table 3

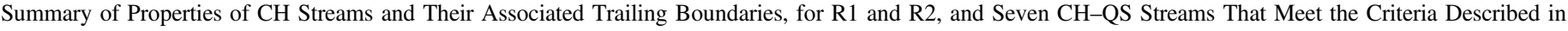
the Text

\begin{tabular}{|c|c|c|c|c|c|c|c|c|c|c|c|c|c|}
\hline Image (UT) & Onset (UT) & $v_{\max }$ & $\mathrm{C}^{6+} / \mathrm{C}^{5+}$ & FIP & $\tau_{\mathrm{CH}}$ & $\tau_{\text {int }}$ & $N_{\text {inv }}$ & $N_{\mathrm{s}}$ & $N_{\text {com }}$ & $A_{\mathrm{C}}$ & $A_{\mathrm{Fe}}$ & $r_{\mathrm{C}}$ & $r_{\mathrm{Fe}}$ \\
\hline $2016 \operatorname{Mar} 25$ (R1) & 2016 Mar 28 & 550 & {$[0.19,1.44]$} & {$[1.7,4.6]$} & 3 & 5.5 & 1 & 3 & 3 & 0.91 & 0.70 & 0.70 & 0.79 \\
\hline 2016 Apr 21 (R2) & 2016 Apr 24 & 550 & {$[0.24,1.22]$} & {$[1.8,4.9]$} & 1 & 6 & 4 & 10 & 5 & 0.34 & 0.22 & 0.46 & $0.23^{*}$ \\
\hline 2016 Jun 30 & 2016 Jul 3 & 470 & {$[0.36,1.4]$} & {$[2.2,6.1]$} & 1.5 & 3.5 & 1 & 4 & 3 & 0.69 & 0.71 & 0.79 & 0.79 \\
\hline 2016 Jul 25 & 2016 Jul 29 & 610 & {$[0.25,1.4]$} & {$[1.4,6.9]$} & 1 & 4.5 & 3 & 6 & 4 & 0.84 & 0.73 & 0.90 & 0.93 \\
\hline 2017 Jan 24 & 2017 Jan 27 & 630 & {$[0.23,1.5]$} & {$[1.4,3.4]$} & 1 & 3.5 & 3 & 5 & 3 & 0.62 & 0.63 & 0.53 & 0.63 \\
\hline 2017 Feb 20 & 2017 Feb 23 & 650 & {$[0.15,1.4]$} & {$[1.7,6.7]$} & 2 & 4 & 3 & 3 & 3 & 0.84 & 0.64 & 0.91 & 0.79 \\
\hline 2017 Mar 19 & 2017 Mar 22 & 700 & {$[0.1,1.5]$} & {$[1.4,6.8]$} & 2.5 & 6 & 4 & 4 & 3 & 0.85 & 0.58 & 0.91 & 0.77 \\
\hline 2017 Aug 1 & 2017 Aug 4 & 690 & {$[0.11,1.4]$} & {$[1.3,5.3]$} & 2 & 7 & 3 & 6 & 4 & 0.87 & 0.85 & 0.79 & 0.89 \\
\hline 2018 Apr 17 & 2018 Apr 20 & 610 & {$[0.16,1.4]$} & {$[1.9,5.8]$} & 2 & 6 & 3 & 7 & 5 & 0.89 & 0.46 & 0.92 & $0.09^{*}$ \\
\hline \multicolumn{2}{|c|}{ Mean (exc. R1, R2) } & 622 & {$[0.2,1.4]$} & {$[1.6,5.9]$} & 1.71 & 4.9 & 2.9 & 5 & 3.6 & 0.80 & 0.66 & 0.82 & 0.70 \\
\hline
\end{tabular}

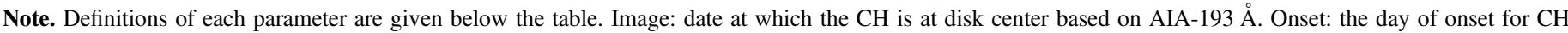

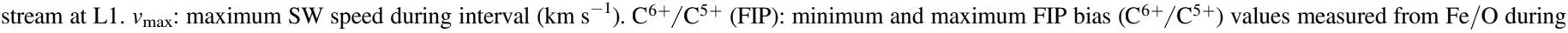

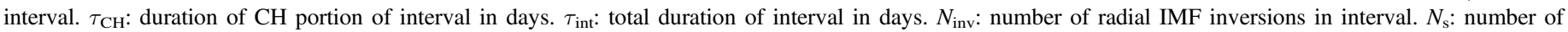

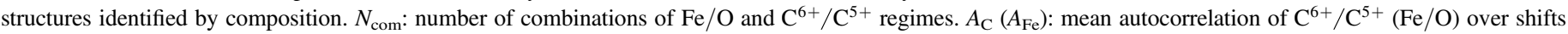

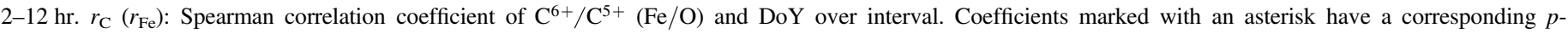
value $>0.01$.

without an intervening fast stream; and (4) no ICMEs (as determined from the ICME list of Richardson \& Cane 2010) or ICME signatures (specifically extended periods of bidirectional strahl) occur between fast wind onset and the HCS. Following these exclusions, seven intervals remain, which we consider to begin at the onset of the fast stream and end at the HCS (this is how R1 and R2 are treated above). While relatively few intervals are identified in comparison to the initial number of candidates, the criteria enforced here ensure that these intervals can be analyzed in an identical manner to R1 and R2.

Table 3 lists some SW properties for the intervals identified in the above selection process. R1, R2, and mean $\mathrm{CH}-\mathrm{QS}$ values are also shown for each parameter. $\mathrm{R} 1$ and $\mathrm{R} 2$ have a below-average maximum speed, a similar range of $\mathrm{C}^{6+} / \mathrm{C}^{5+}$ values, and similar minimum but lower maximum $\mathrm{Fe} / \mathrm{O}$ values to the other intervals. The duration of the $\mathrm{CH}$ stream, $\tau_{\mathrm{CH}}$, is longer in R1 than any of the other intervals, while in R2 it is near shortest. The lengths of the total interval (from onset of the fast stream until HCS) for R1 and R2 fall just above the mean.

Table 3 also lists some derived properties of the $\mathrm{CH}-\mathrm{QS}$ and case study streams. The number of IMF inversions, $N_{\text {inv }}$, over the entire interval is the fewest for R1, while R2 is tied for the most. If $N_{\text {inv }}$ is divided by interval duration, then R2 no longer has the most (although it is still above average). We analyze the composition of the seven $\mathrm{CH}-\mathrm{QS}$ intervals using the same compositional thresholds as were determined above from R1. Comparing to these, $\mathrm{R} 1$ is tied for the lowest number of distinct compositional structures, while $\mathrm{R} 2$ has the most of all intervals. R2 still features the most, even when normalizing $N_{\mathrm{s}}$ by interval duration. The number of combinations of $\mathrm{Fe} / \mathrm{O}$ and $\mathrm{C}^{6+} / \mathrm{C}^{5+}, N_{\mathrm{s}}$, is tied for lowest for $\mathrm{R} 1$ and tied for highest for R2. Examining the time series of $\mathrm{C}^{6+} / \mathrm{C}^{5+}$ for each interval directly, we make a final note that $\mathrm{R} 2$ is the only one to feature $\mathrm{CH}$-like values in a structure that is not adjacent to the main $\mathrm{CH}$ stream.

We perform two additional tests on the compositional trends of these intervals that do not rely on the identification of individual structures using thresholds. In the first, we calculate the autocorrelation function of each $\mathrm{C}^{6+} / \mathrm{C}^{5+}$ and $\mathrm{Fe} / \mathrm{O}$ time series. We list the mean of the autocorrelation $\left(A_{\mathrm{C}}\right.$ and $\left.A_{\mathrm{Fe}}\right)$ over time shifts in the range of $2-12 \mathrm{hr}$ (based on the $2 \mathrm{hr}$
SWICS time resolution) in Table 3. The autocorrelation function for a strongly varying parameter falls off rapidly from 1 (corresponding to a time shift of $0 \mathrm{hr}$ ) as the time shift is increased. $A_{\mathrm{C}}$ and $A_{\mathrm{Fe}}$ thus highlight how variable each parameter is on the chosen timescale. $A_{\mathrm{C}}$ and $A_{\mathrm{Fe}}$ for $\mathrm{R} 2$ are both far from 1 , a result of the variability of $\mathrm{C}^{6+} / \mathrm{C}^{5+}$ and $\mathrm{Fe} / \mathrm{O}$ highlighted in the previous section. For $\mathrm{R} 1$, and in general the other $\mathrm{CH}-\mathrm{QS}$ boundaries, the variation is considerably smoother, and so $A_{\mathrm{C}}$ and $A_{\mathrm{Fe}}$ tend to be closer to 1 .

For the second test, we calculate the Spearman rank-order correlation coefficients of $\mathrm{C}^{6+} / \mathrm{C}^{5+}$ and $\mathrm{Fe} / \mathrm{O}$ against time in each interval and list them in Table $3\left(r_{\mathrm{C}}\right.$ and $\left.r_{\mathrm{Fe}}\right) . r_{\mathrm{C}}$ or $r_{\mathrm{Fe}}=1$ $(-1)$ indicates a perfectly monotonic increase (decrease) with time. Values close to 0 indicate a departure from a monotonic trend. The $p$-values for each coefficient, which indicate the likelihood that uncorrelated data could result in a coefficient of equal or greater magnitude, are vanishingly small in all but two cases. $r_{\mathrm{C}}$ and $r_{\mathrm{Fe}}$ for $\mathrm{R} 1$ and the $\mathrm{CH}-\mathrm{QS}$ boundaries are overall much closer to 1 than for R2 (aside from one outlying case for each ratio). The $\mathrm{CH}-\mathrm{QS}$ boundaries have a similarly monotonic increase in $\mathrm{C}^{6+} / \mathrm{C}^{5+}$ and $\mathrm{Fe} / \mathrm{O}$ to $\mathrm{R} 1$, whereas the increase for $\mathrm{R} 2$ is not large in comparison to the strong variability and so is strongly nonmonotonic as noted above.

\section{Discussion}

\subsection{SW from a $\mathrm{CH}-\mathrm{QS}$ Boundary}

This section evaluates the results for the CH-QS boundary SW during R1 to explain its origins for comparison with the $\mathrm{CH}-\mathrm{AR}$ of R2. We examine the EIS remote sensing results for R1 (Figures 4(a)-(c)) to see whether the R1 in situ compositional configuration can be explained through measurements of relative abundance around the source. Estimates of FIP bias from EIS have been derived using $\mathrm{Si} / \mathrm{S}$ emission, while FIP bias from SWICS is derived using density measurements of $\mathrm{Fe} / \mathrm{O}$. We therefore do not expect direct agreement between these two different FIP bias estimates, as different degrees of fractionation are observed for different elements (e.g., Laming 2011). Discrepancies between remote and in situ abundances have been observed generally (Bochsler 2007). Here, we reasonably expect in situ FIP biases 
that we label high $\mathrm{Fe} / \mathrm{O}$ in Section 2.1 to correspond to enhanced EIS-derived FIP biases of $>2-3$.

We explain the origins of composition observed in situ for $\mathrm{R} 1$ as the $\mathrm{CH}$-labeled portion of the stream in Figure 6 originating in the $\mathrm{CH}$ proper, while the $\mathrm{CHB}$ and QS streams could reasonably originate in locations similar to FIP-enhanced upflowing regions of Boxes 1 and 2 of Figures 4(a)-(c). These locations likely contain closed magnetic field, evidenced by the brightness of emission there (e.g., in Figure 4(a)) and the predicted open flux locations from the PFSS model (Figure 5). Nonpotential open magnetic field, however, cannot be ruled out in these locations, particularly as they lie adjacent to the open $\mathrm{CH}$, and so interchange reconnection may open some of this field. Given these points, in addition to the upflow and enhanced $v_{\mathrm{nt}}$ signatures in these boxes and the presence of strong deflections, and one inversion, of $\phi_{r}$ during the $\mathrm{CHB}$ and QS streams for R1, we conclude that plasma from these types of locations likely escapes into the heliosphere as a result of interchange reconnection. This could take the form of component reconnection (see van Driel-Gesztelyi et al. 2012), as was found in numerous configurations by Fazakerley et al. (2016), owing to the like-polarities of these regions with the adjacent open $\mathrm{CH}$ flux.

Table 3 shows that, relative to comparable $\mathrm{CH}-\mathrm{QS}$ streams, $\mathrm{R} 1$ features few IMF inversions and other possible signatures of reconnection (e.g., in the structuring of compositional features). However, the smoothly varying, monotonically increasing trends of $\mathrm{C}^{6+} / \mathrm{C}^{5+}$ and $\mathrm{Fe} / \mathrm{O}$, consistent with the crossing of a trailing $\mathrm{CH}$ boundary layer (McComas 2003), are present in both R1 and the comparison streams. Differences could be explained by the $\mathrm{CH}-\mathrm{QS}$ boundary for $\mathrm{R} 1$ being especially quiet in terms of interchange reconnection prior to the emergence of AR 12532.

\section{2. $S W$ from a $C H-A R$ Boundary}

We contrast the observations of the $\mathrm{CH}-\mathrm{AR}$ boundary in $\mathrm{R} 2$ with results from the equivalent $\mathrm{CH}-\mathrm{QS}$ boundary in $\mathrm{R} 1$, which reveals clear differences in the durations of equivalent in situ streams. We also contrast R2 with observations of the seven example $\mathrm{CH}-\mathrm{QS}$ streams to give further context. However, we note that whereas it is reasonable that the main driver of change between R1 and R2 should be the emergence of AR 12532 (based on the results of the remote observations and backmapping), many factors likely influence differences between R1/R2 and these example streams that are associated with a different $\mathrm{CH}$. The $\mathrm{CH}$ stream in both $\mathrm{R} 1$ and $\mathrm{R} 2$ (also labeled S1 for R2 in Figure 7) was found to persist for $\sim 3$ days in $\mathrm{R} 1$ (longer than for any of the comparable $\mathrm{CH}-\mathrm{QS}$ intervals in Table 3 ) but only $\sim 1.2$ days in $\mathrm{R} 2$ (similar to the briefest $\mathrm{CH}$ in the comparison intervals). It is possible that in $\mathrm{R} 2$ there is more open magnetic flux associated with source regions of non- $\mathrm{CH}$-composition plasma than there is in $\mathrm{R} 1$. In $\mathrm{R} 2$, this would both decrease the size of the $\mathrm{CH}$-composition stream and increase the size of the non- $\mathrm{CH}$-composition streams that follow it. This is consistent with open magnetic flux being transferred from the $\mathrm{CH}$ to previously closed locations through interchange reconnection between the $\mathrm{CH}$ and the $\mathrm{AR}$. If this is the case, then the emergence of AR 12532 here drives a change in $\mathrm{CH}$ stream duration from the longest of all $\mathrm{CH}-\mathrm{QS}$ streams considered here to near the shortest.

The CHB and QS periods in R1 are both around 1 day in length, while in R2 S2-S10 each last around 8-16 hr. The values of $\mathrm{C}^{6+} / \mathrm{C}^{5+}$ and $\mathrm{Fe} / \mathrm{O}$ in these structures do not increase monotonically, instead fluctuating between values characteristic of different sources. This contrasts strikingly with $\mathrm{R} 1$ and most of the seven example $\mathrm{CH}-\mathrm{QS}$ boundaries, where these ratios increase relatively smoothly. The mean number of structures for these intervals, however, falls somewhere between $\mathrm{R} 1$ and $\mathrm{R} 2$. The five different combinations of $\mathrm{Fe} / \mathrm{O}$ and $\mathrm{C}^{6+} / \mathrm{C}^{5+}$ values in structures of $\mathrm{R} 2$ suggest at least five distinct sources, as opposed to the three suggested in R1. This is consistent with the increased occurrence of interchange reconnection, releasing plasma of different properties to the heliosphere. The changes in composition could thus be produced by the opening of distinct loops, likely of a range of sizes or at different heights (Section 2.3). Again, the mean number of combinations for the $\mathrm{CH}-\mathrm{QS}$ examples is intermediate between R1 and R2. R1 appears to be an initially nonactive $\mathrm{CH}-\mathrm{QS}$ boundary, heavily altered by the emergence of AR 12532. Although the effects of this emergence are not so dramatic as to produce compositions that are unique to a $\mathrm{CH}-$ AR boundary, the overall compositional structure and evolution of R2 (particularly the variability and departure from a monotonic increase) is clearly distinct from average $\mathrm{CH}-\mathrm{QS}$ boundary properties.

R2 features more large $\left(\geqslant 45^{\circ}\right)$ azimuthal deviations in IMF from the Parker spiral angle than R1 during the periods of interest, four of which constitute inversions relative to the radial direction. Similar to the above case for composition, the example $\mathrm{CH}-\mathrm{QS}$ intervals are somewhat intermediate between $\mathrm{R} 1$ and $\mathrm{R} 2$ in the occurrence of these inversions. We interpret these deviations/inversions as kinks in the IMF due to reconnection, which we favor over waves and turbulence, due to the tendency for these deflections to align with compositional structures (Section 2.3). The $\mathrm{CH}-\mathrm{AR}$ boundary in this case study thus shows evidence of increased interchange reconnection relative in particular to the preceding $\mathrm{CH}-\mathrm{QS}$ case of $\mathrm{R} 1$ and also to the mean comparable $\mathrm{CH}-\mathrm{QS}$ boundary.

Across the $\mathrm{S} 2-\mathrm{S} 10$ portion of $\mathrm{R} 2, \mathrm{C}^{6+} / \mathrm{C}^{5+}$ and $\mathrm{Fe} / \mathrm{O}$ measurements never exceed their upper limits as measured during R1. This is not entirely unexpected, given the extent of compositional overlap for AR and QS SW (Kilpua et al. 2016; Fu et al. 2017). EIS observations in Figures 4(a)-(f) both reveal localized enhancements of FIP bias, which could reasonably correspond to the enhancements found in $\mathrm{Fe} / \mathrm{O}$ for both rotations. A greater enhancement in FIP bias (of 3.4 in Box 2; Section 3.3) was found at the source for R1 than for R2. We do not necessarily expect a corresponding greater $\mathrm{Fe} / \mathrm{O}$ to be present in situ during R1, since EIS FIP measurements are made at only a few locations. The mean upper limits of FIP biases found in the seven example $\mathrm{CH}-\mathrm{QS}$ streams in Table 3 are higher than those for $\mathrm{R} 1$ and $\mathrm{R} 2$, emphasizing that the presence of an AR at the source does not guarantee an increase in FIP bias of the resulting SW.

We now turn to the possible origins of the SW structures S1S11 during R2. From the mapping, we default to the $\mathrm{CH}$ and $\mathrm{CH}-\mathrm{AR}$ boundary being the most likely region for sources of the observed SW and only consider the AR or other sources if strong evidence exists in in situ-remote sensing comparisons. $\mathrm{S} 1, \mathrm{~S} 6$, and $\mathrm{S} 8$ are structures with $\mathrm{CH}$-like composition. While we are confident that $\mathrm{S} 1$ originates in the $\mathrm{CH}$ proper, this is less certain for S6 and S8, as they fall between clearly non-CH streams, a feature not present in any of the $\mathrm{CH}-\mathrm{QS}$ intervals. This could be a result of numerous interchange reconnection 
processes, occurring because of the presence of AR 12532, leading to open flux that is rooted in a $\mathrm{CH}$ source region being interspersed with flux that is predominantly rooted outside of the $\mathrm{CH}$.

We consider periods with $\mathrm{CH}$-like $\mathrm{Fe} / \mathrm{O}$ and non-CH-like $\mathrm{C}^{6+} / \mathrm{C}^{5+}$ values: $\mathrm{S} 2, \mathrm{~S} 4$, and S5. These are similar to the composition of a CH boundary from McComas et al. (2002). Such plasma may originate from regions such as Box 4 of Figures 4(d)-(f), which exhibit low/moderate FIP bias, but are likely to exhibit enhanced charge state, as they fall outside of the $\mathrm{CH}$ itself.

There are four structures of high $\mathrm{Fe} / \mathrm{O}$ and intermediate or high $\mathrm{C}^{6+} / \mathrm{C}^{5+}: \mathrm{S} 3, \mathrm{~S} 7, \mathrm{~S} 9$, and S10. Their sources possess both higher electron temperatures and FIP bias than CHs. One explanation for these structures is that they may originate from around the $\mathrm{CH}$ boundary, at a region of enhanced FIP bias of 2-3. One such region is evident in Box 3 of Figures 4(d)-(f), which also coincides with strong upflow and enhanced $v_{\mathrm{nt}}$.

As we have suggested candidate sources on the $\mathrm{CH}$ boundary (such as Boxes 3 and 4 of Figures 4(d)-(f)) for both of the above compositional signatures, we note that smaller regions of enhanced upflow are found along the boundary. The co-observation of IMF kinks and deflections means that interchange reconnection is a viable explanation for how plasma might escape from all of these candidate sources into the SW.

There is some evidence that plasma escapes to the SW from near AR 12532 itself; in Box 5, there are strong upflows (Figure 4(h)) and nearby open flux (Figure 5). This indicates that plasma from this region of the AR is likely able to escape into the SW through interchange reconnection. However, since this open flux is rooted far to the north of the SW sourcepoints, it appears unlikely that this plasma would make its way to the ecliptic to be observed by $A C E$. Chained reconnection is a possible mechanism by which this plasma could escape into the SW at $A C E$ latitudes. However, this remains unconfirmed, as plasma from the AR does not appear to be necessary to explain the composition of SW for R2. Given the evidence available here, it remains ambiguous whether the SW in structure $\mathrm{S} 11$ is associated with the AR or not. However, from the above arguments, that does not appear likely either.

\subsection{Results in Context of AR SW Mechanisms}

Multiple pieces of evidence (from composition, IMF orientation, and structuring and duration of streams) have been identified for interchange reconnection between the $\mathrm{CH}$ - and AR-associated loops being responsible for the non- $\mathrm{CH} \mathrm{SW}$ during R2. This reconnection conceivably occurs across a range of heights, with loops of different sizes and/or properties, and releases plasma from multiple regions of distinct composition. It is unsurprising that this is the primary explanation for the observed SW, given that this observational period was chosen because of the emergence of an AR adjacent to a $\mathrm{CH}$, an ideal source of open flux for interchange reconnection.

There are no clear instances where only loop expansion could explain any of the structures S1-10 during R2. The typical in situ signature of a closed loop, bidirectional strahl, does not appear during R2. Loop expansion may, however, still be taking place, as loops might disconnect at one end before reaching $1 \mathrm{au}$, or extend far enough into the heliosphere that strahl from one of the footpoints is highly broadened (e.g., as described by Crooker \& Owens 2012). Loop expansion may also be releasing plasma, e.g., from near the AR, which misses $A C E$. The former case may be partially responsible for the kinked IMF signatures described in Section 3.4. However, given the evidence listed above for interchange reconnection as a source mechanism, we cannot conclude that loop expansion makes as significant a contribution to the SW here.

For R2, it is difficult to unambiguously infer chained reconnection processes as an SW mechanism because more evidence is suggestive of interchange reconnection. Since interchange reconnection seems plausible here in a range of configurations, it appears that plasma could travel along multiple reconnected field lines while making its way into the heliosphere. Thus, although only one instance of interchange reconnection is strictly required to open a given loop to the heliosphere and explain the observations made here, multiple steps might take place. This is not the compelling case for this mechanism made by Culhane et al. (2014) and Mandrini et al. (2014), who found one AR to be entirely covered by a helmet streamer, and so AR plasma could reach the SW only through multiple steps of reconnection.

\section{Conclusions}

In this study, we have isolated the effects of an AR on the SW produced from the trailing edge of a $\mathrm{CH}$. We have done so by contrasting in situ and remote sensing observations between two consecutive Carrington rotations, occurring before and after the emergence of AR 12532. We conclude through ballistic backmapping techniques and remote sensing observation that the primary source of variation in the SW between the two rotations is the influence of AR 12532. This study thus allows the isolation of AR influence on the SW from other effects in a manner that is distinct from previous work. Our results show that the emergence of an AR to the east of a $\mathrm{CH}$ significantly influences the nature of the SW from the initially inactive $\mathrm{CH}$ and $\mathrm{CHB}$. These results have strong implications for source region identification for $\mathrm{CH}$ SW that is modulated by an AR.

The effects of AR 12532 on the SW originating from the $\mathrm{CH}$ boundary are primarily on the structure and composition of the wind. While the initial CH-QS boundary produces SW that can be separated into three distinct periods (probably from two or three distinct source regions, which we find typical for most example $\mathrm{CH}-\mathrm{QS}$ streams), the $\mathrm{CH}-\mathrm{AR}$ boundary here leads to many more (10 or 11) compositional structures, of variable composition, manifesting in situ, with at least five distinct origins responsible for them. They are also associated, although not perfectly coincident, with strong deviations in the magnetic field from its expected orientation in both azimuth and elevation. The variability of composition, resulting from this structuring, reveals the $\mathrm{CH}-\mathrm{AR}$ boundary to be distinct from the broadly smooth and monotonic compositional evolution that we find typical of $\mathrm{CH}-\mathrm{QS}$ boundaries (although the individual composition and IMF features are not fully exclusive to $\mathrm{CH}-\mathrm{AR}$ streams). Considering this in situ evidence and EIS observations that allow us to diagnose properties and processes at the source region, we conclude that the structural and compositional changes following the emergence of AR 12532 are most consistent with increased instances of interchange reconnection. This reconnection occurs at the $\mathrm{CH}-\mathrm{AR}$ boundary, and possibly other locations, between AR-associated loops and $\mathrm{CH}$ open flux. There is no in situ evidence to uniquely suggest that the SW we observe in this study at L1 
necessarily originates near the core of the AR. We recommend the extension of this work with a statistical study, to determine whether the above features, here attributed to reconnection, are universally more common in $\mathrm{CH}-\mathrm{AR}$ than $\mathrm{CH}-\mathrm{QS}$ boundaries. The seven example $\mathrm{CH}-\mathrm{QS}$ boundaries collected here provide a suitable starting point for such work.

We find no conclusive evidence for the loop expansion and chained reconnection processes described in Section 1. These may be occurring but are not readily identifiable in this configuration where interchange reconnection should dominate. To isolate and test these mechanisms, we suggest further study of unique AR SW configurations such as this one, chosen to be most likely to isolate the process of interest. In particular, future observations from Solar Orbiter and Parker Solar Probe will be ideal for such studies. At lower heliocentric distances, SW stream properties are expected to more closely reflect source region properties, allowing more reliable identification of SW sources. Further, signatures of the source mechanism, such as bidirectional strahl and inverted IMF flux, may also become more prominent. The in situ observations from both of these spacecraft, and the remote sensing and composition observations from Solar Orbiter in particular, thus promise to greatly enhance our understanding of the influence of ARs on the SW.

The authors thank the Wind-3DP, ACE-SWEPAM, ACE-MAG, $A C E$-SWICS, SDO-AIA, and SDO-HMI teams for provision of data in this study. We acknowledge use of NASA/GSFC's Space Physics Data Facility's CDAWeb service to access $A C E$ and Wind data (https://cdaweb.sci.gsfc.nasa.gov). SDO-AIA and $S D O$-HMI data are courtesy of NASA/SDO and the AIA, EVE, and HMI science teams (available at https://sdo.gsfc.nasa.gov/ data). Hinode is a Japanese mission developed and launched by ISAS/JAXA, with NAOJ as domestic partner and NASA and UKSA as international partners. It is operated by these agencies in cooperation with ESA and NSC (Norway). Figures 3, 6, and 7 were produced using the Matplotlib plotting library for Python (Hunter 2007). We thank Marc De Rosa for useful discussions regarding PFSS software. A.R.M. was supported by the Science and Technology Facilities Council (STFC) through $\mathrm{PhD}$ studentship to UCL/MSSL ST/N504488/1 and consolidated grant to University of Reading ST/R000921/1. C.J.O., D.B., L. K.H., and R.T.W. are supported by STFC consolidated grants to UCL/MSSL, ST/N00722/1, and ST/S000240/1. The work of D.H.B. was performed under contract to the Naval Research Laboratory and was funded by the NASA Hinode program. D. M.L. is grateful to STFC for the award of an Ernest Rutherford Fellowship (ST/R003246/1).

\section{ORCID iDs}

Allan R. Macneil (1) https://orcid.org/0000-0003-4802-1209 Christopher J. Owen 주 https://orcid.org/0000-0002-5982-4667 Deborah Baker (1) https://orcid.org/0000-0002-0665-2355 David H. Brooks (1) https://orcid.org/0000-0002-2189-9313 Louise K. Harra (1) https://orcid.org/0000-0001-9457-6200 David M. Long (1) https://orcid.org/0000-0003-3137-0277 Robert T. Wicks (ib https://orcid.org/0000-0002-0622-5302

\section{References}

Antiochos, S., DeVore, C., Karpen, J., \& Mikić, Z. 2007, ApJ, 671, 936 Antiochos, S., Mikić, Z., Titov, V., Lionello, R., \& Linker, J. 2011, ApJ, 731,112
Asplund, M., Grevesse, N., Sauval, A., \& Scott, P. 2009, ARA\&A, 47, 481 Baker, D., Brooks, D., Démoulin, P., et al. 2013, ApJ, 778, 69

Baker, D., Brooks, D. H., van Driel-Gesztelyi, L., et al. 2018, ApJ, 856, 71 Baker, D., Rouillard, A. P., van Driel-Gesztelyi, L., et al. 2009, AnGeo, 27,3883

Baker, D., van Driel-Gesztelyi, L., \& Attrill, G. 2007, AN, 328, 773

Bame, S., Asbridge, J., Feldman, W., \& Gosling, J. 1977, JGR, 82, 1487

Bochsler, P. 2007, A\&ARv, 14, 1

Brooks, D., \& Warren, H. 2011, ApJL, 727, L13

Brooks, D. H., Ugarte-Urra, I., \& Warren, H. P. 2015, NatCo, 6, 5947

Bruno, R., \& Carbone, V. 2013, LRSP, 10, 2

Crooker, N., Gosling, J., \& Kahler, S. 2002, JGRA, 107, 1028

Crooker, N., Kahler, S., Larson, D., \& Lin, R. 2004, JGRA, 109, 3108

Crooker, N., \& Owens, M. 2012, SSRv, 172, 201

Culhane, J., Brooks, D., van Driel-Gesztelyi, L., et al. 2014, SoPh, 289, 3799

Culhane, J., Harra, L., James, A., et al. 2007, SoPh, 243, 19

Del Zanna, G., Aulanier, G., Klein, K.-L., \& Török, T. 2011, A\&A, 526, A137

Del Zanna, G., Dere, K. P., Young, P. R., Landi, E., \& Mason, H. E. 2015, A\&A, 582, A56

Dere, K., Landi, E., Mason, H., Fossi, B. M., \& Young, P. 1997, A\&AS, 125, 149

Doschek, G., Warren, H., Mariska, J., et al. 2008, ApJ, 686, 1362

Edwards, S., Parnell, C., Harra, L., Culhane, J., \& Brooks, D. 2016, SoPh, 291, 117

Fazakerley, A., Harra, L., \& van Driel-Gesztelyi, L. 2016, ApJ, 823, 145

Feldman, U., Widing, K., \& Warren, H. 1999, ApJ, 522, 1133

Fisk, L. 2003, JGRA, 108, 1157

Fisk, L., Schwadron, N., \& Zurbuchen, T. 1998, SSRv, 86, 51

Fox, N., Velli, M., Bale, S., et al. 2016, SSRv, 204, 7

Fu, H., Li, B., Li, X., et al. 2015, SoPh, 290, 1399

Fu, H., Madjarska, M., Xia, L., et al. 2017, ApJ, 836, 169

Geiss, J., Gloeckler, G., \& Von Steiger, R. 1995, SSRv, 72, 49

Gloeckler, G., Geiss, J., Balsiger, H., et al. 1992, A\&AS, 92, 267

Gopalswamy, N., Mäkelä, P., Akiyama, S., et al. 2013, SoPh, 284, 17

Gosling, J., Baker, D., Bame, S., et al. 1987, JGRA, 92, 8519

Gosling, J., Skoug, R., McComas, D., \& Smith, C. 2005, JGRA, 110, 1107

Grevesse, N., Asplund, M., \& Sauval, A. J. 2007, SSRv, 130, 105

Harra, L., Matthews, S., \& Culhane, J. 2001, ApJL, 549, L245

Harra, L., Sakao, T., Mandrini, C., et al. 2008, ApJL, 676, L147

Heidrich-Meisner, V., Peleikis, T., Kruse, M., Berger, L., \& Wimmer-Schweingruber, R. 2016, A\&A, 593, A70

Huang, Z., Frazin, R., Landi, E., et al. 2012, ApJ, 755, 86

Hundhausen, A., Gilbert, H., \& Bame, S. 1968, ApJL, 152, L3

Hunter, J. D. 2007, CSE, 9, 90

Kashyap, V., \& Drake, J. J. 2000, BASI, 28, 475

Kepko, L., Viall, N., Antiochos, S., et al. 2016, GeoRL, 43, 4089

Kilpua, E., Madjarska, M., Karna, N., et al. 2016, SoPh, 291, 2441

Ko, Y.-K., Muglach, K., Wang, Y.-M., Young, P., \& Lepri, S. 2014, ApJ, 787, 121

Kojima, M., Fujiki, K., Ohmi, T., et al. 1999, JGR, 104, 16993

Kosugi, T., Matsuzaki, K., Sakao, T., et al. 2007, The Hinode Mission (Berlin: Springer), 5

Krieger, A., Timothy, A., \& Roelof, E. 1973, SoPh, 29, 505

Krista, L. D., \& Gallagher, P. T. 2009, SoPh, 256, 87

Laming, J. M. 2012, ApJ, 744, 115

Laming, J. M., Drake, J., \& Widing, K. 1995, ApJ, 443, 416

Lemen, J., Akin, D., Boerner, P., et al. 2011, The Solar Dynamics Observatory (Berlin: Springer), 17

Lin, R., Anderson, K., Ashford, S., et al. 1995, SSRv, 71, 125

Lockwood, M., Owens, M. J., \& Macneil, A. 2019, SoPh, 294, 85

Mandrini, C., Nuevo, F., Vasquez, A., et al. 2014, SoPh, 289, 4151

McComas, D. 2003, AIP Conf. Proc. 679 in Solar Wind Ten (Melville, NY: AIP), 33

McComas, D., Bame, S., Barker, P., et al. 1998, in The Advanced Composition Explorer Mission, ed. C. T. Russell, R. A. Mewaldt, \& T. T. Von Rosenvinge (Springer, Netherlands: Dordrecht), 563

McComas, D., Elliott, H., \& von Steiger, R. 2002, GeoRL, 29, 1314

Meyer, J.-P. 1985, ApJS, 57, 173

Montgomery, M. D., Asbridge, J., Bame, S., \& Feldman, W. 1974, JGR, 79,3103

Morgan, H., Jeska, L., \& Leonard, D. 2013, ApJS, 206, 19

Müller, D., \& St. Cyr, O.C. 2013, Proc. SPIE., 8862, 88620E

Neugebauer, M., Forsyth, R., Galvin, A., et al. 1998, JGRA, 103, 14587

Neugebauer, M., Liewer, P., Smith, E., Skoug, R., \& Zurbuchen, T. 2002, JGRA, 107, 1488

Nolte, J., \& Roelof, E. 1973, SoPh, 33, 483

Ogilvie, K., \& Desch, M. 1997, AdSpR, 20, 559 
Owens, M., Crooker, N., \& Lockwood, M. 2013, JGRA, 118, 1868

Owens, M. J., Lockwood, M., Barnard, L. A., \& MacNeil, A. R. 2018, ApJL, 868, L14

Owocki, S., Holzer, T., \& Hundhausen, A. 1983, ApJ, 275, 354

Parker, E. 1988, ApJ, 330, 474

Pesnell, W. 2015, Solar Dynamics Observatory (SDO) (Berlin: Springer)

Pierrard, V., Maksimovic, M., \& Lemaire, J. 2001, Ap\&SS, 277, 195

Pilipp, W., Miggenrieder, H., Montgomery, M., et al. 1987, JGRA, 92, 1093

Priest, E., \& Démoulin, P. 1995, JGRA, 100, 23443

Richardson, I., \& Cane, H. 2010, SoPh, 264, 189

Riley, P., Linker, J., Mikić, Z., et al. 2006, ApJ, 653, 1510

Riley, P., \& Lionello, R. 2011, SoPh, 270, 575

Riley, P., \& Luhmann, J. 2012, SoPh, 277, 355

Rosner, R., Tucker, W., \& Vaiana, G. 1978, ApJ, 220, 643

Sakao, T., Kano, R., Narukage, N., et al. 2007, Sci, 318, 1585

Schatten, K., Wilcox, J., \& Ness, N. 1969, SoPh, 6, 442

Scherrer, P., Schou, J., Bush, R., et al. 2012, SoPh, 275, 207
Schrijver, C., \& DeRosa, M. 2003, SoPh, 212, 165

Schwadron, N., Fisk, L., \& Zurbuchen, T. 1999, ApJ, 521, 859

Schwadron, N., McComas, D., Elliott, H., et al. 2005, JGRA, 110, 4104

Schwenn, R. 2007, Solar Dynamics and Its Effects on the Heliosphere and Earth (Berlin: Springer), 51

Schwenn, R. 1990, in Large-Scale Structure of the Interplanetary Medium, ed. R. Schwenn \& E. Marsch (Berlin: Springer), 99

Smith, C., LH'eureux, J., Ness, N., et al. 1998, The Advanced Composition Explorer Mission (Berlin: Springer), 613

Stone, E., Frandsen, A., \& Mewaldt, R. 1998, SSRv, 86, 1

Uchida, Y., McAllister, A., Strong, K., et al. 1992, PASJ, 44, L155

van Driel-Gesztelyi, L., Culhane, J., Baker, D., et al. 2012, SoPh, 281, 237

Verbeeck, C., Delouille, V., Mampaey, B., \& De Visscher, R. 2014, A\&A, 561, A29

Wang, Y.-M., Hawley, S. H., \& Sheeley, N. R. 1996, Sci, 271, 464

Wang, Y.-M., \& Sheeley, N., Jr 1990, ApJ, 355, 726

Zhao, L., Zurbuchen, T., \& Fisk, L. 2009, GeoRL, 36, L14104 